\title{
UCRL-TR-232789
}

LAWRENCE LIVERMORE NATIONAL LABORATORY

\section{Beam and Truss Finite Element Verification for DYNA3D}

Howard J. Rathbun

July 18, 2007 
This document was prepared as an account of work sponsored by an agency of the United States Government. Neither the United States Government nor the University of California nor any of their employees, makes any warranty, express or implied, or assumes any legal liability or responsibility for the accuracy, completeness, or usefulness of any information, apparatus, product, or process disclosed, or represents that its use would not infringe privately owned rights. Reference herein to any specific commercial product, process, or service by trade name, trademark, manufacturer, or otherwise, does not necessarily constitute or imply its endorsement, recommendation, or favoring by the United States Government or the University of California. The views and opinions of authors expressed herein do not necessarily state or reflect those of the United States Government or the University of California, and shall not be used for advertising or product endorsement purposes.

This work was performed under the auspices of the U.S. Department of Energy by University of California, Lawrence Livermore National Laboratory under Contract W-7405-Eng-48. 


\begin{abstract}
The explicit finite element (FE) software program DYNA3D has been developed at Lawrence Livermore National Laboratory (LLNL) to simulate the dynamic behavior of structures, systems, and components. This report focuses on verification of beam and truss element formulations in DYNA3D. An efficient protocol has been developed to verify the accuracy of these structural elements by generating a set of representative problems for which closed-form quasi-static steady-state analytical reference solutions exist. To provide as complete coverage as practically achievable, problem sets are developed for each beam and truss element formulation (and their variants) in all modes of loading and physical orientation. Analyses with loading in the elastic and elastic-plastic regimes are performed. For elastic loading, the FE results are within $1 \%$ of the reference solutions for all cases. For beam element bending and torsion loading in the plastic regime, the response is heavily dependent on the numerical integration rule chosen, with higher refinement yielding greater accuracy (agreement to within 1\%). Axial loading in the plastic regime produces accurate results (agreement to within $0.01 \%$ ) for all integration rules and element formulations. Truss elements are also verified to provide accurate results (within $0.01 \%$ ) for elastic and elastic-plastic loading. A sample problem to verify beam element response in ParaDyn, the parallel version DYNA3D, is also presented.
\end{abstract}




\section{TABLE OF CONTENTS}

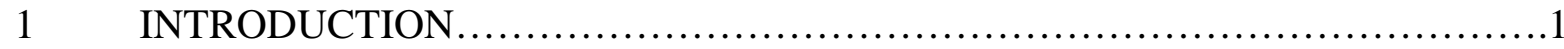

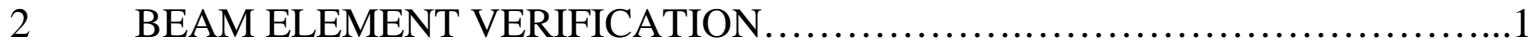

2.1 BEAM ELEMENT FORMULATIONS ....................................

2.2 OVERVIEW OF VERIFICATION METHODOLOGY...........................2

$2.3 \quad$ ELASTIC RESPONSE.......................................................

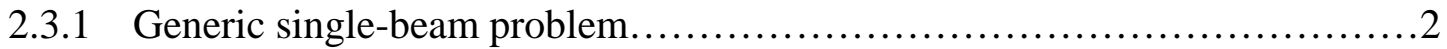

2.3.2 Representative Load cases...................................................

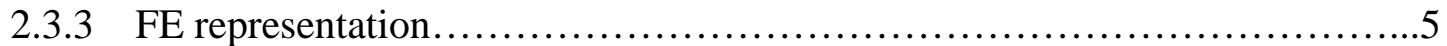

2.3.3.1 Load curves......................................................... 7

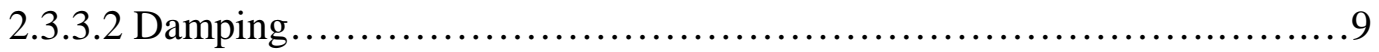

2.3.3.3 Anticipated response....................................................

2.3.3.4 Generation of the DYNA3D input decks..............................

2.3.3.5 Extraction of information..............................................9

2.3.3.6 Convergence study............................................. 10

2.3.4 Analysis and results......................................................

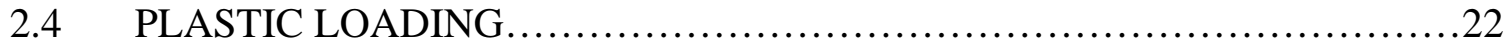

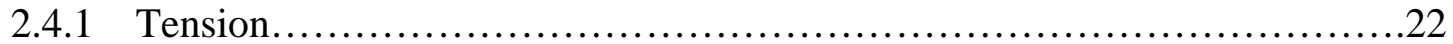

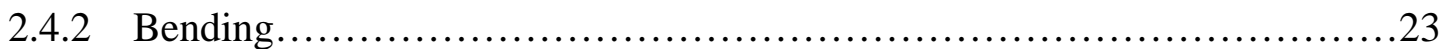

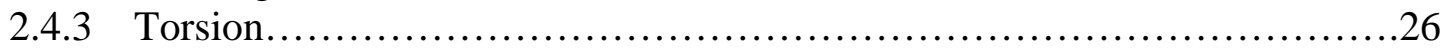

2.4.4 Thin-walled tube with Tension / Torsion Loading..........................27

2.5 UNI-AXIAL ELASTIC DEFORMATION - PARALLEL PROBLEM...........30

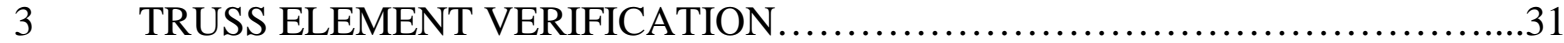

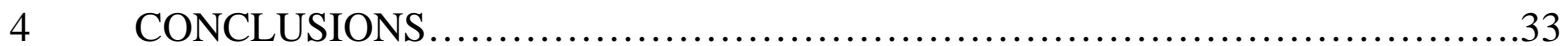

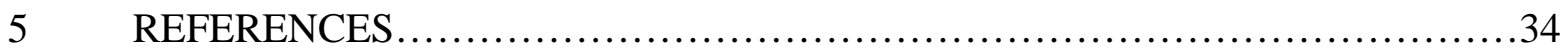

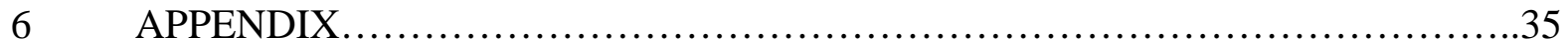




\title{
Beam and Truss Finite Element Verification for DYNA3D
}

\author{
Howard J. Rathbun \\ Advanced Engineering Analysis Group \\ Defense Technologies Engineering Division
}

\section{INTRODUCTION}

The explicit finite element (FE) code DYNA3D has been developed at Lawrence Livermore National Laboratory (LLNL) to simulate the dynamic behavior of solids and structures [1]. To ensure the accuracy of DYNA3D results, verification and validation efforts are underway within the Advanced Simulation and Computing program at LLNL. In this context, verification involves basic tests to determine that the element formulations are implemented as desired. This objective can be attained using relatively simple test problems that correspond to closed-form analytical reference solutions that are consistent with the element formulation. Validation efforts generally involve more complex models and compare the results of simulations to analytical or experimental results that are representative of a problem of interest. The analytical results used for validation are not, in general, derived using the same assumptions or conditions as those used to derive the element formulation.

The current study focuses on verification of beam and truss elements in DYNA3D. First, we present some basic beam problems for which analytical reference solutions exist. We then develop FE models, which are grouped into problem sets, and compare their results with the analytical solutions. We examine beam element responses for all orientations and modes of loading using both elastic and elastic-plastic material models (constrained to the elastic range). We also discuss the elastic-plastic responses of single beam elements loaded in tension, bending, and torsion. The final beam element verification problems involve the tension-torsion elasticplastic response of a thin-walled hollow tube beam element, and a many-element problem, designed for future use to verify the parallel implementation of DYNA3D beam elements. Finally, truss element verification is presented.

\section{BEAM ELEMENT VERIFICATION}

\subsection{BEAM ELEMENT FORMULATIONS}

DYNA3D offers many options for beam elements; users will choose options based upon the geometry, loading, boundary conditions, and material properties of the structure being analyzed. The two major categories of beam element formulations, named for their initial developers, are: Hughes-Liu (H-L) [2,3] and Belytschko-Schwer (B-S) [4,5]. Variants of the H-L formulation are based on the type of numerical integration performed across the element cross-section:

- User-defined numerical integration (H-L0)

- 2x2 Gauss quadrature (H-L2)

- $3 \times 3$ Gauss quadrature (H-L3) 
- 3x3 Lobotto quadrature (H-L4)

- $4 \times 4$ Gauss quadrature (H-L5)

Note that, since B-S elements are resultant-based, no numerical integration is performed across the cross section. Including both B-S and $\mathrm{H}-\mathrm{L}$ formulations, there are six beam element variants: B-S, H-L0, H-L2, H-L3, H-L4, and H-L5.

Each element formulation can be used with elastic or elastic-plastic material models, allowing the user flexibility to model a wide range of structural behaviors. Material models 1 (elastic), 3 (kinematic/isotropic elastic-plastic) and 24 (rate-dependent tabular isotropic elastic-plastic) are available for $\mathrm{H}$-L elements and material models 1,3 , and 28 (resultant plasticity) are available for B-S elements.

Each beam element is defined by two nodes (plus a third node used only to orient the local coordinate system), each having three translational and three rotational degrees of freedom. Beam elements can be aligned in any orientation in three-dimensional space. They can be loaded by applying axial or transverse forces, moments, or torques. They may also be loaded by prescribing axial, transverse, rotational, or torsional displacements.

\subsection{OVERVIEW OF VERIFICATION METHODOLOGY}

Given this wide array of modeling options, an efficient protocol for evaluating the accuracy of element performance is required. The verification methodology begins by identifying representative problems in which a load is applied to a single beam, and one or more reference solutions to the problem are identified. The solutions involve either reactions or deflections that the beam is expected to experience, given its material properties and boundary conditions. Next, FE models are generated that simulate each reference problem. The analyses are completed in DYNA3D, and the results are compared to the reference solutions. This facilitates a quantitative evaluation of the accuracy of the beam elements as implemented in the code.

\subsection{ELASTIC RESPONSE}

Elastic response is probed using a collection of simple beam problems grouped into individual problem sets that encompass all of the FE beam variants described in section 2.1. The geometry and material properties of the beams and the imposed loads are selected so that the displacements are small and the beams remain within the elastic regime even when elastic-plastic material models are employed. This allows direct comparisons with available analytical solutions.

\subsubsection{Generic single-beam problem}

We define a generic problem using a beam that is cantilevered at one end. Consider the beam shown in Figure 1. The reference axes $i, j$, and $k$ refer to the beam's local coordinate system. 


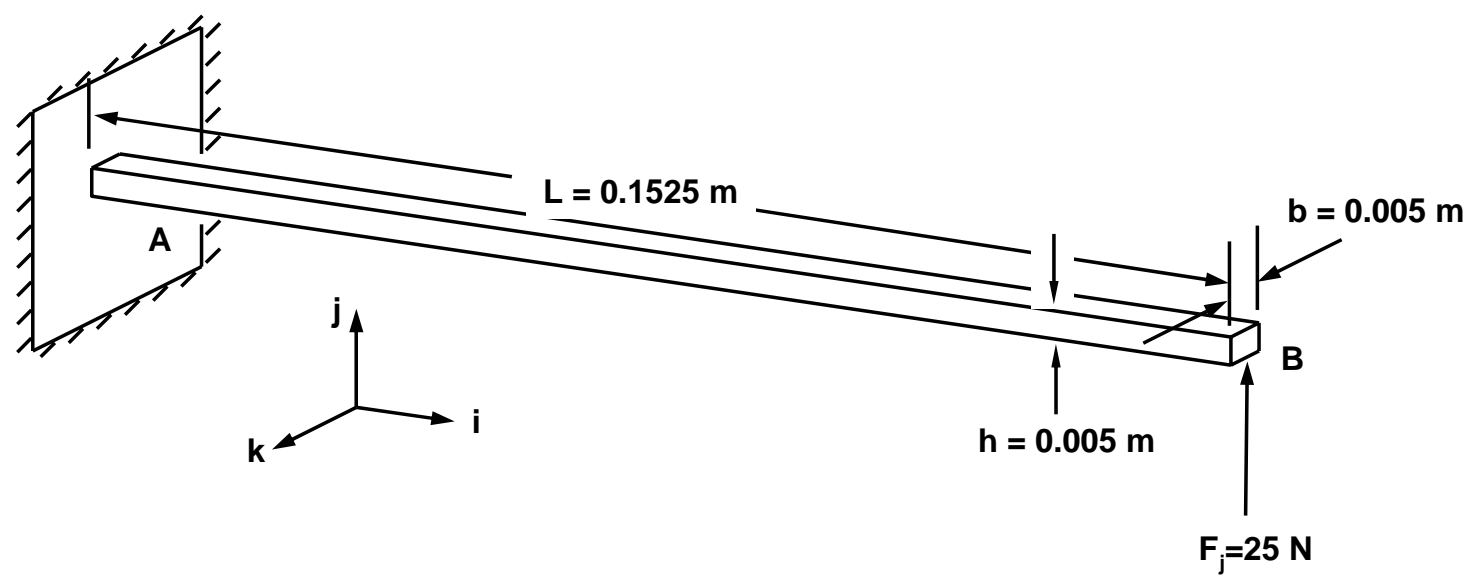

Figure 1. Beam geometry for verification problems

The response of the beam to various loads can be described in closed-form. Consider, for example, a transverse shear load, $F_{j}$, applied at end B in the $j$-direction as shown in Figure 1. The resulting deflection, $u_{j}$, at end $\mathrm{B}$ is given by [6]:

$$
u_{j}=\frac{F_{j} L^{3}}{3 E I_{k}}
$$

where $L$ is the length, $E$ is the elastic modulus, and $I_{k}$ is the moment of inertia about the $k$-axis given by:

$$
I_{k}=\frac{b h^{3}}{12}
$$

$b$ and $h$ are the width and height, respectively. The rotation with respect to the k-axis, $\phi_{k}$, at end $\mathrm{B}$ is given by [6]:

$$
\phi_{k}=\frac{F_{j} L^{2}}{2 E I_{k}}
$$

Equations 1 and 3 are reference solutions for the single-beam problem shown in Figure 1.

\subsubsection{Representative Load cases}

The generic single-beam problem described above is expanded to explore a variety of load cases. With reference to Figure 1, loads that can be applied to end B include: 
- axial force

- transverse force

- bending moment / torque

- axial translation

- transverse translation

- rotation

Table 1 lists six load cases in which a force or moment is applied, and corresponding reference solutions for translation and rotation at end $\mathrm{B}$. In Table $1, M$ is an applied moment, $A_{c}$ is the cross-sectional area, and $J$ is the polar moment of inertia. Note that a force applied in a direction other than the primary (i) axis of the beam creates transverse shear, and a moment about the primary axis of the beam creates torsional loading. Table 2 lists six load cases in which a translation or rotation is applied and their reference solutions. In Table 2, $G$ is the shear modulus. For these load cases, the reaction at end A and the rotation or translation at end B are chosen as reference solutions. While there are other reference solutions, for example the reaction at end A for load cases listed in Table 1, those listed represent a practical and reasonably thorough set to accomplish the verification goals established in this report. The load magnitudes are chosen such that the beam's deflection is small relative to its length, thus ensuring that the reference solutions are valid.

Table 1. Applied Force/Moment, Boundary Conditions and Reference Solutions

\begin{tabular}{|c|c|c|c||}
\hline \hline Load Case Number & Applied Force/Moment at End B & End B Translation & End B Rotation \\
\hline \hline 1 & $F_{i}=1000 N$ & $u_{i}=\frac{F_{i} L}{A_{c} E}$ & $\phi_{k}=0$ \\
\hline 2 & $F_{j}=25 N$ & $u_{j}=\frac{F_{j} L^{3}}{3 E I_{k}}$ & $\phi_{k}=\frac{F_{j} L^{2}}{2 E I_{k}}$ \\
\hline 3 & $F_{k}=25 N$ & $u_{k}=\frac{F_{k} L^{3}}{3 E I_{j}}$ & $\phi_{j}=\frac{F_{k} L^{2}}{2 E I_{j}}$ \\
\hline 4 & $M_{i}=4 N \cdot m$ & 0 & $\phi_{i}=\frac{M_{i} L}{J_{i} G}$ \\
\hline 5 & $M_{j}=2.5 N \cdot m$ & $u_{k}=\frac{M_{j} L^{2}}{2 E I_{j}}$ & $\phi_{j}=\frac{M_{j} L}{E I_{j}}$ \\
\hline 6 & $M_{k}=2.5 N \cdot m$ & $u_{j}=\frac{M_{k} L^{2}}{2 E I_{k}}$ & $\phi_{k}=\frac{M_{k} L}{E I_{k}}$ \\
\hline
\end{tabular}


Table 2. Applied Translation/Rotation, Boundary Conditions and Reference Solutions

\begin{tabular}{|c|c|c|c||}
\hline Load Case Number & $\begin{array}{l}\text { Applied Translation / } \\
\text { Rotation at End B }\end{array}$ & Reaction at End A & $\begin{array}{l}\text { End B Rotation / } \\
\text { Translation }\end{array}$ \\
\hline \hline 7 & $u_{i}=3.05 \times 10^{-5} \mathrm{~m}$ & $F_{i}=\frac{A_{c} E}{L} u_{i}$ & 0 \\
\hline 8 & $u_{j}=2.836 \times 10^{-3} m$ & $F_{j}=\frac{3 E I_{k}}{L^{3}} u_{j}, M_{k}=\frac{3 E I_{k}}{L^{2}} u_{j}$ & $\phi_{k}=\frac{3}{2 L} u_{j}$ \\
\hline 9 & $u_{k}=2.836 \times 10^{-3} m$ & $F_{k}=\frac{3 E I_{j}}{L^{3}} u_{k}, M_{j}=\frac{3 E I_{j}}{L^{2}} u_{k}$ & $\phi_{j}=\frac{3}{2 L} u_{k}$ \\
\hline 10 & $\phi_{i}=0.078 \mathrm{rad}$ & $M_{i}=\frac{J_{i} G}{L} \phi_{i}$ & 0 \\
\hline 11 & $\phi_{j}=0.0366 \mathrm{rad}$ & $M_{j}=\frac{E I_{j}}{L} \phi_{j}$ & $u_{k}=\frac{L}{2} \phi_{j}$ \\
\hline 12 & $\phi_{k}=0.0366 \mathrm{rad}$ & $M_{k}=\frac{E I_{k}}{L} \phi_{k}$ & $u_{j}=\frac{L}{2} \phi_{k}$ \\
\hline
\end{tabular}

\subsubsection{FE representation}

Figure 2 shows the basic FE model used to simulate each beam load case. The model consists of five collinear elements. Node 1 , at end A, is held fixed in translation and rotation, and a load or displacement is applied to node 6 at end B. FE beam models are developed for each of the twelve load cases in Tables 1 and 2. The length, $L$, of each beam is $0.1525 \mathrm{~m}$, and the crosssection is square with height, $h$, and width, $b$, both equal to $0.005 \mathrm{~m}$. The beam has a density of $7860 \mathrm{~kg} / \mathrm{m}^{3}$.

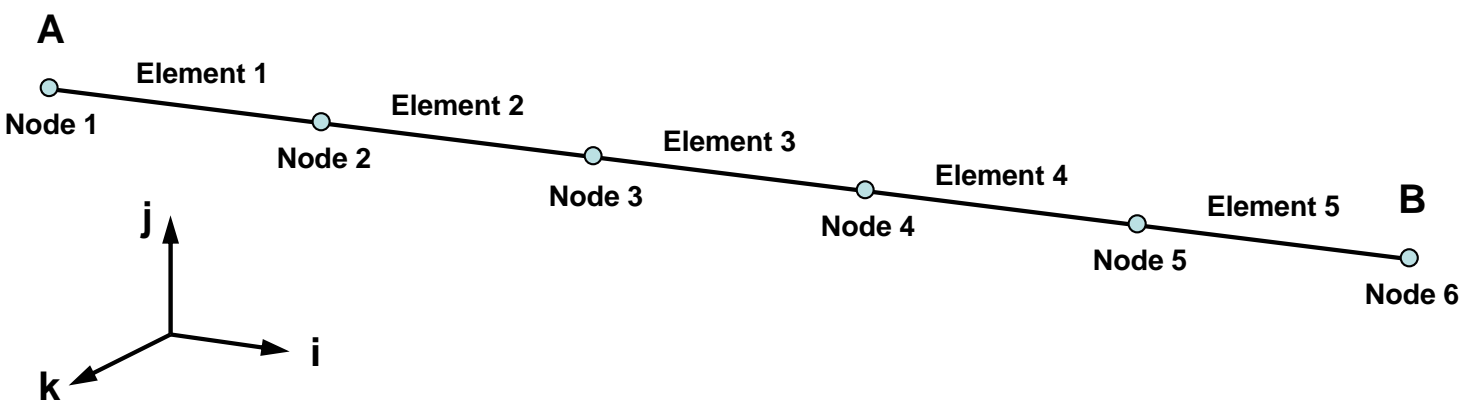

Figure 2. Five-element FE model of beam geometry 
Beam elements can assume any orientation in three-dimensional space. To provide assurance that the accuracy of the DYNA3D formulations does not depend on beam or load orientation, we consider three groups of beam models that are oriented in x-, y-, and z-directions, respectively. These groups, illustrated in Figures 3-5, respectively, depict the consolidated load cases that form a single problem set for beams oriented in each of the three global directions. Specifically, twelve beam models in three orientations results in 36 separate models in a problem set. For example, in load case 1 for the x-oriented beam models (denoted 1-x in Figure 3), an axial load, $F_{x}$, is applied at end B. Similarly, in load case 9 for the z-oriented beam models (denoted 9-z in Figure 5), a transverse displacement, $u_{y}$, is applied at end B.

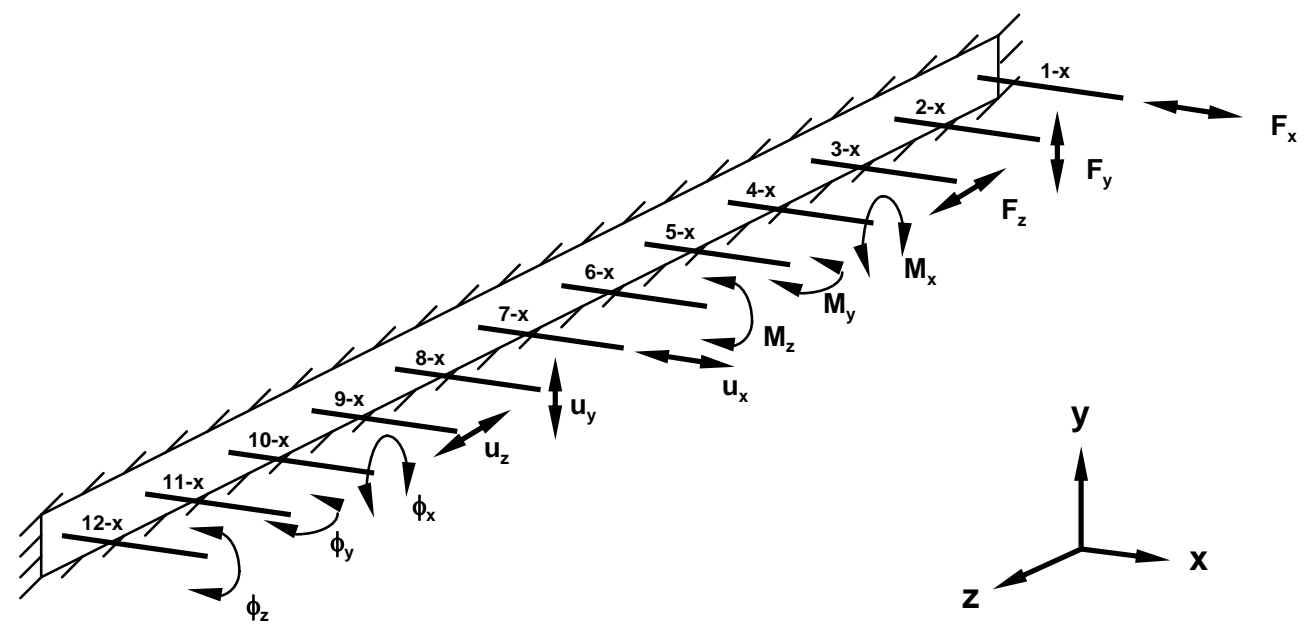

Figure 3. x-oriented beams with imposed "free-end" boundary conditions

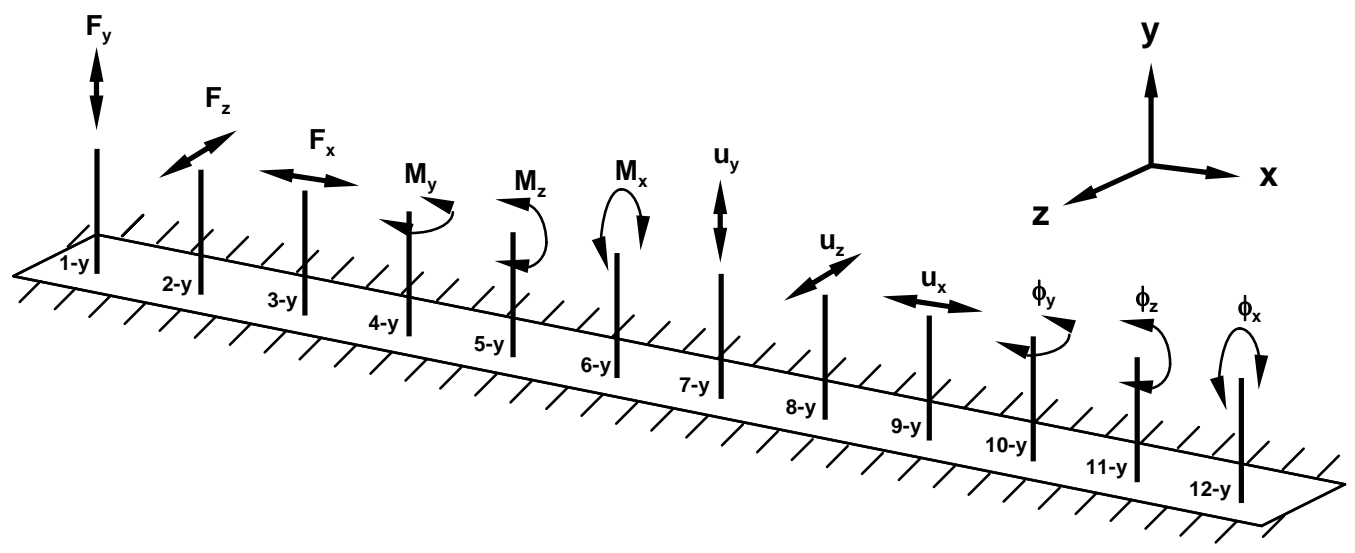

Figure 4. y-oriented beams with imposed "free-end" boundary conditions 


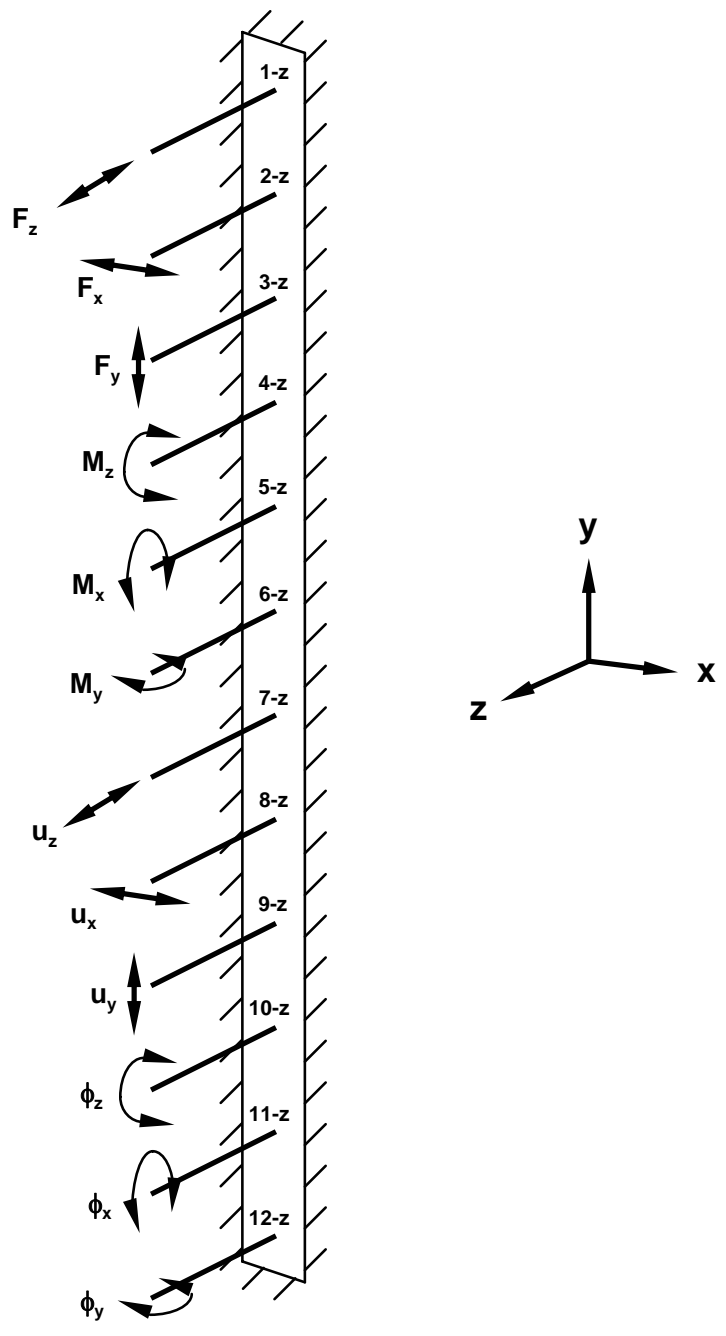

Figure 5. z-oriented beams with imposed "free-end" boundary conditions

\subsubsection{Load curves}

In DYNA3D, the time dependence of an applied load is prescribed by a load curve. Figures 6 and 7 show the load curves defined for the load cases of Tables 1 and 2, respectively, i.e., the conditions in Table 1 are generated using the load curve in Figure 6, and the conditions in Tble 2 are generated using the load curve in Figure 7. Note that, in lieu of direct prescription, the displacements listed in Table 2 are obtained by specifying nodal velocities through the load curve shown in Figure 7. In each case, the applied force, moment, translational or rotational velocity is scaled by the magnitude of the appropriate load curve. 
For example, in the first load case of Table 1, an axial force of $1000 \mathrm{~N}$ is applied at end $\mathrm{B}$ through the load curve shown in Figure 6. Thus, the axial load is linearly increased from 0 to $1000 \mathrm{~N}$ over a time of 0.015 seconds, held constant at that magnitude until 0.075 seconds, and then linearly reduced to 0 at 0.9 seconds. The process is then repeated for compression by applying the remaining negative-amplitude portion of the curve.

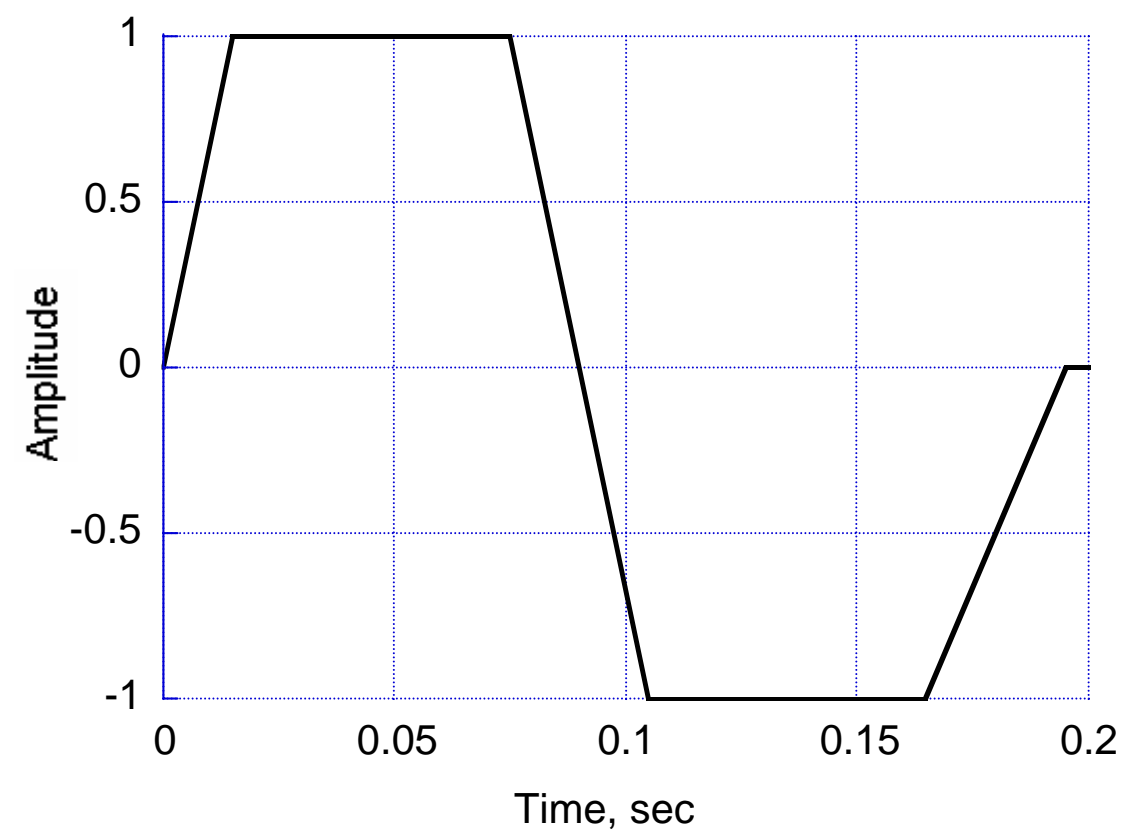

Figure 6. Load curve time history for Table 1 load cases

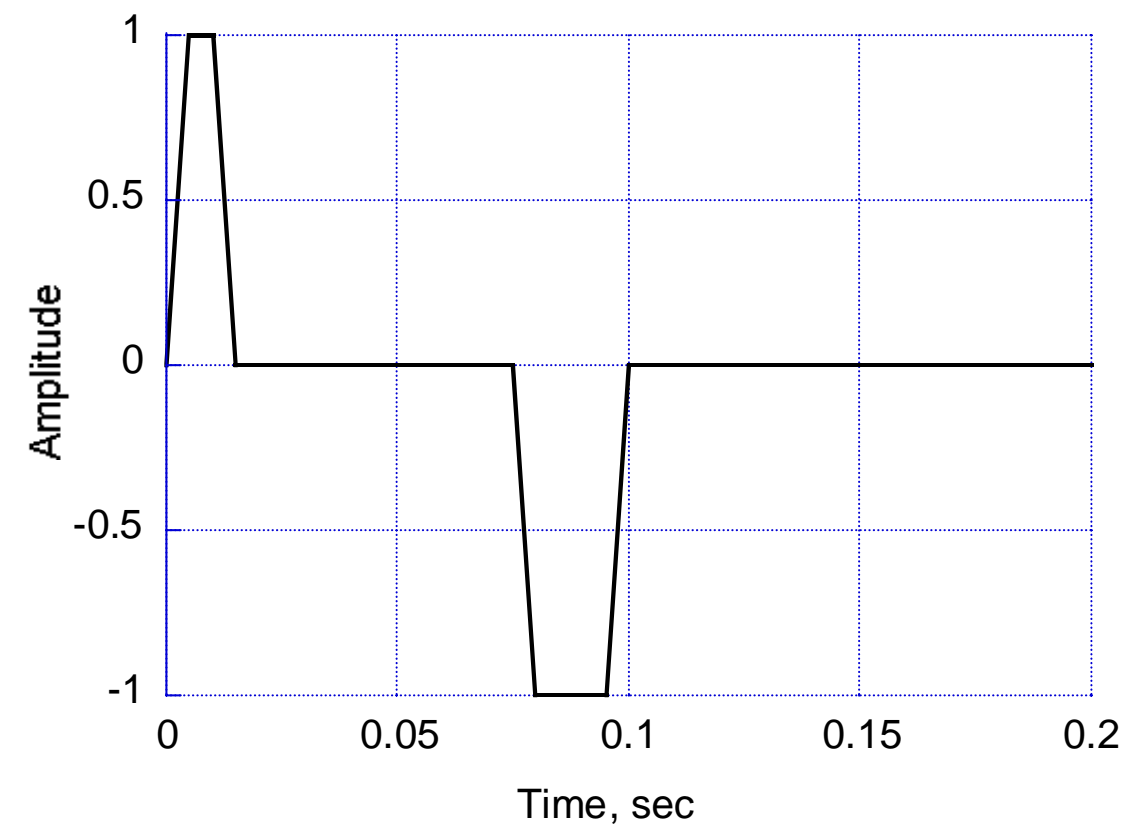

Figure 7. Load curve time history for Table 2 load cases 
As a second example, in the first load case of Table 2, an axial displacement of $3.05 \times 10^{-5} \mathrm{~m}$ is applied at end B. To achieve this displacement, a nodal velocity of $3.05 \times 10^{-3} \mathrm{~m} / \mathrm{s}$ is applied to end $\mathrm{B}$ of the beam and amplified by the load curve in Figure 7. The period of zero velocity between 0.015 and 0.075 sec corresponds to a positive steady-state axial displacement. Between 0.075 and $0.1 \mathrm{sec}$, the nodal velocity is reversed to create a compressive displacement of the same magnitude, and held constant for the duration of the analysis.

The beam element verification problem sets discussed thus far are quasi-static in nature. To achieve quasi-static loading in DYNA3D and ensure that inertial effects do not significantly influence the results, the load curves are defined over a time scale that is much longer than the structural response time.

\subsubsection{Damping}

As presently defined, the FE beam models produce a response that oscillates about the steadystate deflection. To simplify comparison between the reference solution and the steady-state DYNA3D response, the oscillation is attenuated by prescribing mass-proportional damping [1] with parameters $\xi=1.0$ and $\omega=200 \mathrm{rad} / \mathrm{sec}$. These values are chosen to allow the beam to reach a steady state in a reasonable period of time without overly restraining the response.

\subsubsection{Anticipated response}

Because the load curve scales the applied load, the beam's response should roughly follow the load curve's temporal dependence. As an example, in load case 2 of Table 1, a transverse force is applied at end B through the load curve shown in Figure 6. Hence, the translation, $u_{j}$, and rotation, $\phi_{k}$, are expected to initially increase and reach an upper plateau, then reverse and reach a lower plateau, then return to zero. Some initial ringing is expected when the load history reaches the plateaus, followed by attenuation to steady state due to the mass-proportional damping.

\subsubsection{Generation of the DYNA3D input decks}

The FE problem sets are generated using the commercial mesh generation software TrueGrid [7]. Each five-element beam is an individual part, with the appropriate boundary conditions and loading applied at ends A and B. As an example, the TrueGrid input file for linear elastic verification problems is included in the Appendix. TrueGrid processes the input file (which contains the geometry, loading and boundary conditions, and element and material properties) and generates the DYNA3D input deck. Certain necessary features are added manually to prepare the DYNA3D input deck, including [1] requests for element time history output, MILI plot output format, exact output time, rotational velocity and displacement output, and massproportional damping.

\subsubsection{Extraction of information}

DYNA3D records output data in three files: a high-speed print (HSP) file, a force (FORRCT) file, and a plot (PLT) file. The HSP and FORRCT files are in text format and store stress, displacement, and force data that will be used for future automated regression testing. The PLT 
file is a binary file that is read by the visualization software program Griz [8], which is used to extract time history information for displacements, forces, and moments for the verification parameters of interest. In load cases 7-12, force and/or moment information at end A is required. This information is not available in the PLT file so DYNA3D was prompted to output this information in the corresponding FORRCT file.

\subsubsection{Convergence study}

A convergence study is performed to assess the sensitivity of DYNA3D beam elements to mesh density. Certain loading configurations, such as transverse bending, can produce results that are highly dependent on mesh density; axial and torsional loading results are less dependent. In the current study, beam models are subjected to a transverse load at end B while end A was held fixed in translation and rotation. FE models are generated, using from one to twenty elements to represent the beam. The material properties and loading are identical to that described in Section 2.1. Both B-S and H-L3 beam element variants are considered. Two simulation types are run:

i. An un-damped simulation in which the free vibration natural frequency is recorded

ii. A simulation with mass-proportional damping to check the steady-state displacement response

Figure 8 shows the un-damped beam natural frequency as a function of the number of elements for both the B-S and H-L3 element variants. The reference solution is given by [9]:

$$
\omega_{n}=(\beta L)^{2} \sqrt{\frac{E I}{\rho A L^{4}}}
$$

where $\beta L=1.875$ for the first mode of vibration. The observed natural frequency increases with the number of elements for both element formulations.

Figure 9 shows the damped, steady-state transverse displacement for both B-S and H-L3 beams as a function of the number of elements. In this case, the B-S element provides a highly accurate solution, regardless of the number of elements, whereas the H-L3 result is highly meshdependent. Based on the results in Figures 8 and 9, five elements appear to give reasonable agreement with the reference solutions (to within $0.96 \%$ in displacement and $4.72 \%$ in frequency) and, therefore, the FE models used for this validation study include five elements.

\subsubsection{Analysis and results}

Problem sets are developed for each of the six beam element variants using material model 1 (linear elastic). A separate DYNA3D input deck is generated for each problem set. To simplify generation of the input deck, 3x3 Gauss quadrature is employed as the user-defined integration rule for the H-L0 variant. Four additional problem sets are developed to study elastic response using elastic-plastic material models. In this group, H-L2 elements are used with material models 3 and 24 and B-S elements are used with material models 3 and 28. 


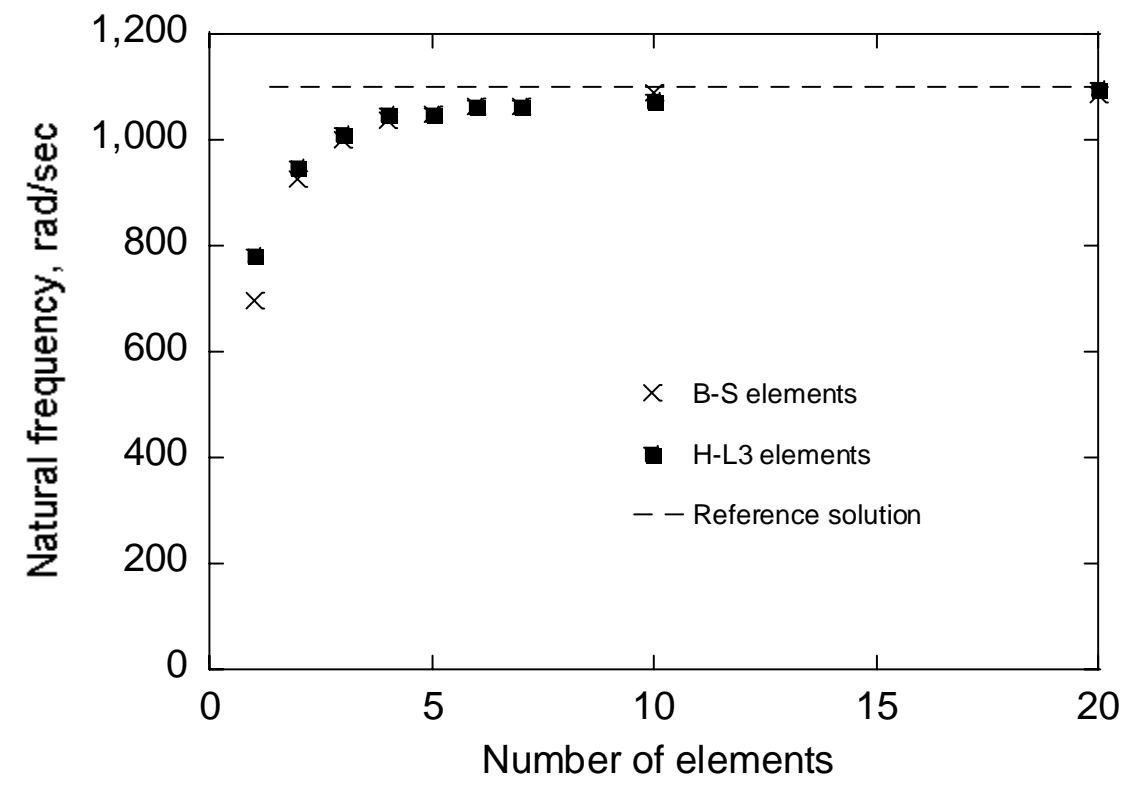

Figure 8. Un-damped natural frequency vs. number of beam elements for transverse loading.

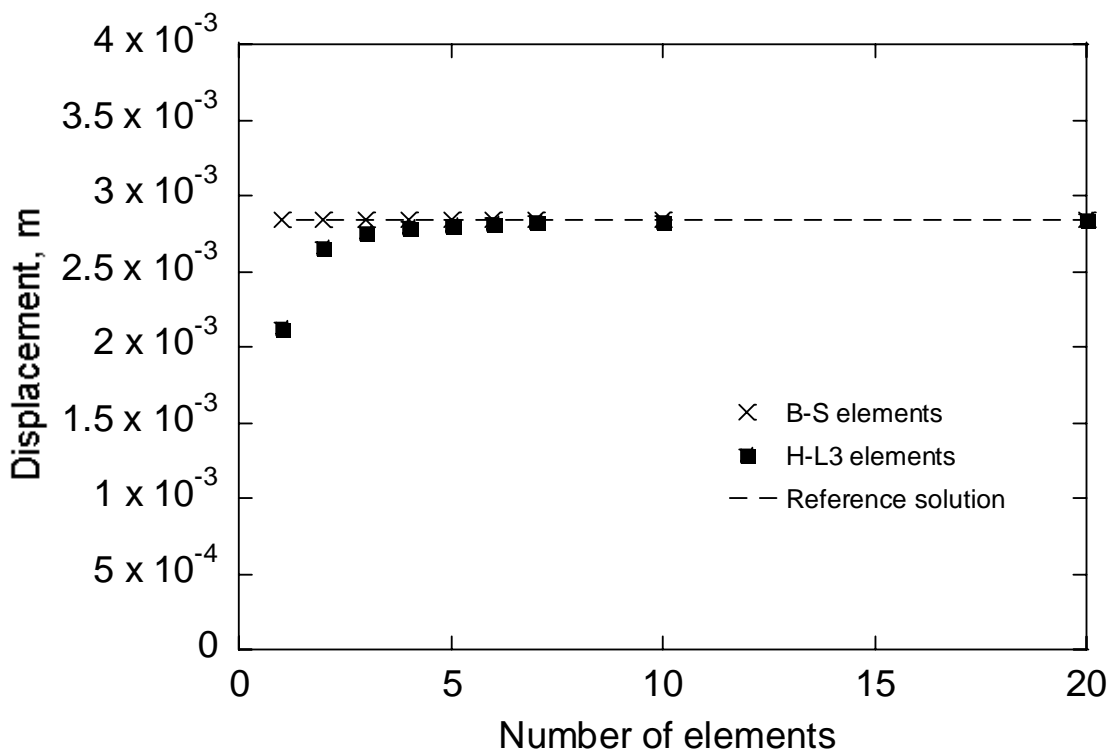

Figure 9. Damped displacement vs. number of beam elements for transverse loading.

Note that when a user specifies material model 3 for B-S beams, DYNA3D internally calls material model 28. However, DYNA3D employs the material model 3 interface and this needs to 
be verified. The yield strength, $\sigma_{y}$, is chosen such that the response remains elastic. Table 3 lists the material properties used in these analyses. The beam element verification problem sets have been analyzed using DYNA3D Version 6.1 (developmental), compiled on 03/02/2006.

Table 3. Material properties for elastic response problems

\begin{tabular}{|c|c|c|c|c|c|c||}
\hline Material model & $E, \mathrm{GPa}$ & $v$ & $\rho, \mathrm{kg} / \mathrm{m}^{3}$ & $\sigma_{y}, \mathrm{MPa}$ & $E_{t}, \mathrm{GPa}$ & $\beta$ \\
\hline \hline 1 & 200 & 0.3 & 7860 & N/A & N/A & N/A \\
\hline 3 & 200 & 0.3 & 7860 & 250 & $\approx 0$ & 1 \\
\hline 24 & 200 & 0.3 & 7860 & 250 & $\approx 0$ & N/A \\
\hline 28 & 200 & 0.3 & 7860 & 250 & $\approx 0$ & N/A \\
\hline
\end{tabular}

The solid curve in Figure 10 shows the axial displacement, $u_{i}$, at end B of Beam 1-x as a function of time for the B-S beam element type. This analysis corresponds to load case 1 (Table 1) with the prescribed load history shown in Figure 6. The displacement magnitude follows the general trajectory of the prescribed load curve. The displacement initially increases, then levels off to the steady-state tensile maximum. Next, the displacement decreases and levels off at the steadystate compressive minimum. Finally, when the load is released, the displacement returns to zero.

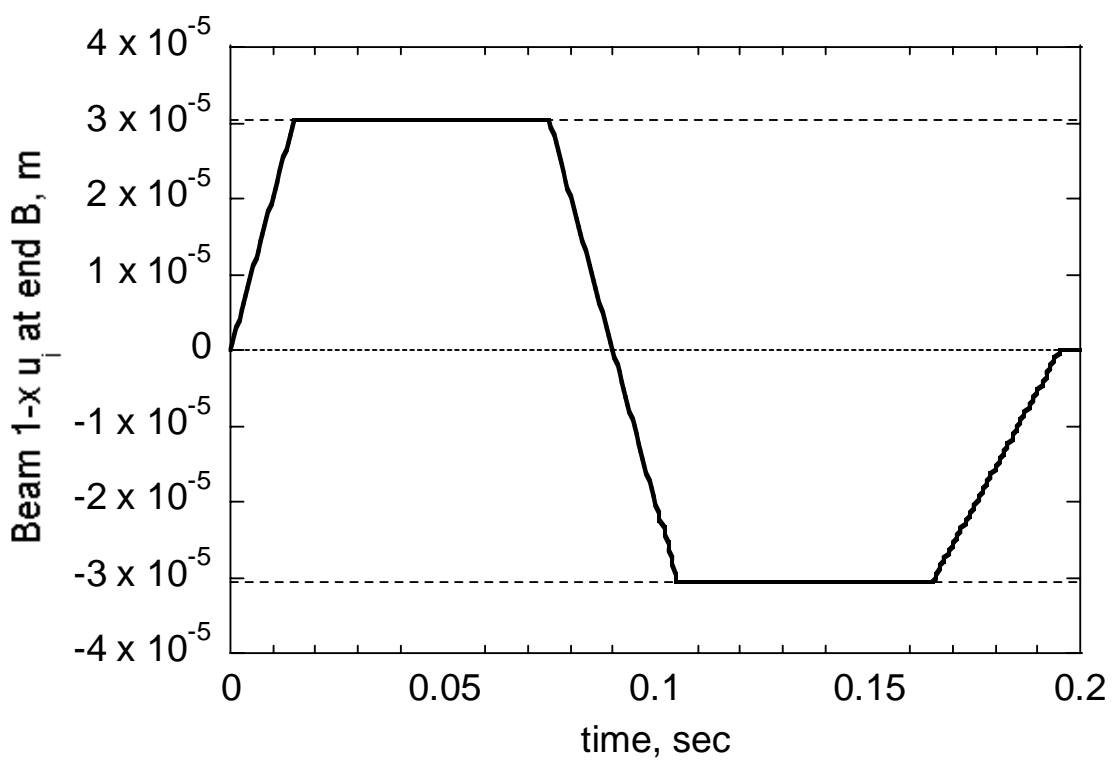

Figure 10. Axial displacement for beam 1-x at end B vs. time. 
The dotted lines in Figure 10 indicate the reference solution, for both tensile and compressive loading. There is $0.000 \%$ difference between the DYNA3D and reference solutions for this case, where percent difference is defined by:

$$
\% \text { difference }=\text { abs }((D Y N A 3 D \text { result }- \text { reference solution }) / \text { reference solution }) \times 100
$$

For this load case, the rotational response of Beam 1-x at end B is identically zero about all three coordinate axes.

The solid curve in Figure 11 shows the transverse displacement response, $u_{j}$, at end B of Beam 2$\mathrm{x}$ as a function of time for the B-S beam element type, corresponding to load case 2 in Table 1. The dotted lines in Figure 11 give the reference solution for loading in both the positive and negative $j$-directions. The slight overshoot seen in Figure 11 is an inertial effect, and the small vibration about the steady-state solution is attenuated through mass proportional damping. In this case, there is a $0.009 \%$ difference between the DYNA3D steady-state displacements and the reference solutions. Figure 12 shows the rotation, in radians, at end B for the B-S beam model for load case 2 . In this case, the steady-state difference from the reference solution is $0.063 \%$.

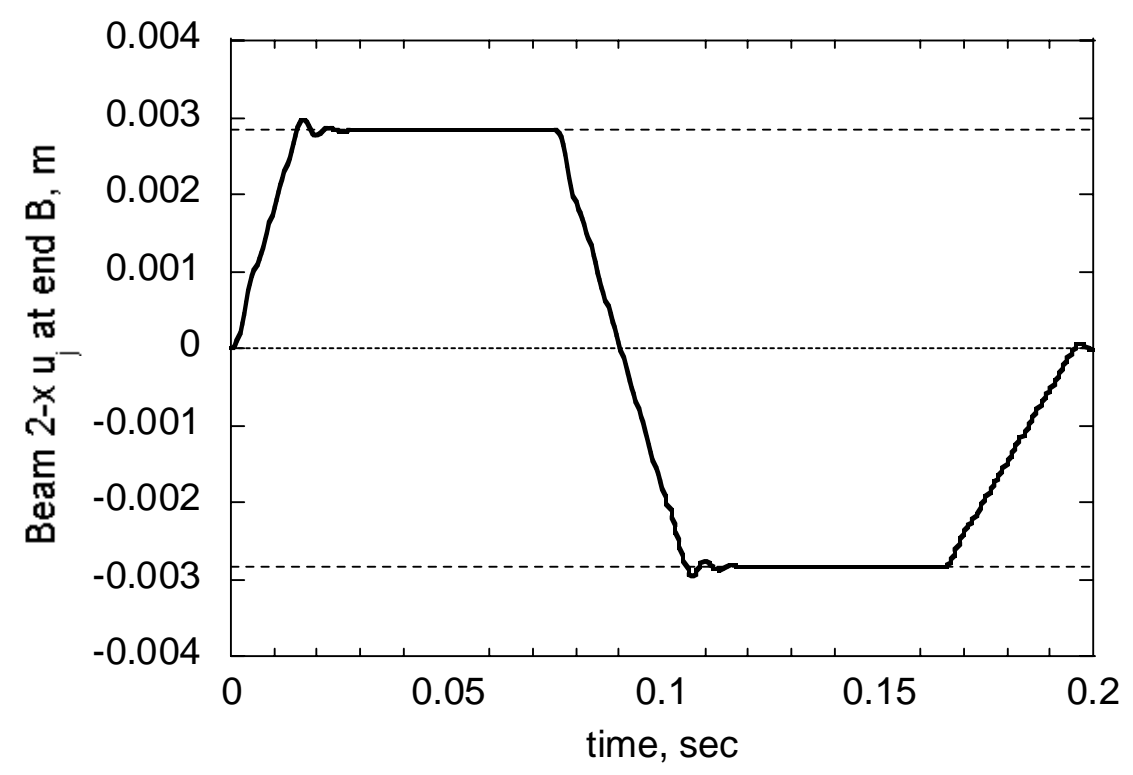

Figure 11. Transverse displacement for beam 2-x at end B vs. time. 


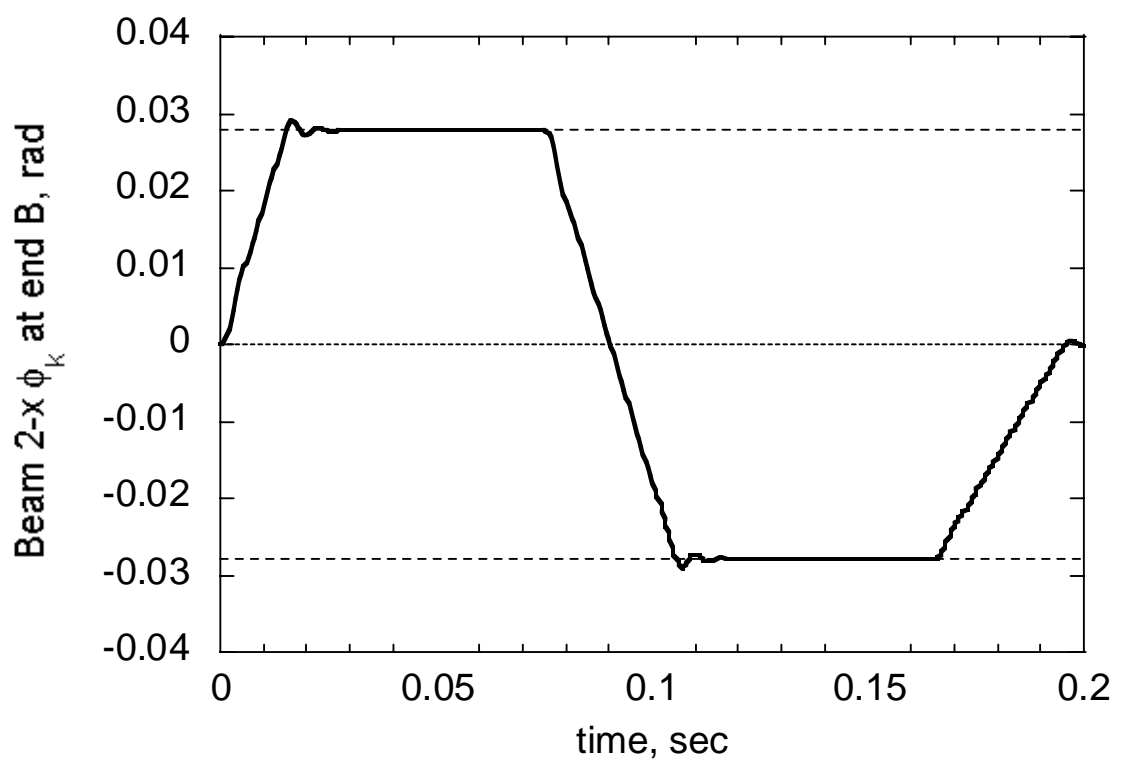

Figure 12. Rotation for beam 2-x at end B vs. time.

Referring to Table 1, load case 3 is directly analogous to load case 2, the only difference being the direction of loading. The results of load case 3 have been verified to be numerically equivalent to load case 2; hence, load case 3 is considered redundant to load case 2. Similarly, load cases 6, 9, and 12 are redundant to load cases 5, 8 and 11, respectively. In the interest of brevity, only the non-redundant load case results are presented in Figures 13-22 (Note that all of the load cases were analyzed in order to comfirm redundancy). All load cases demonstrate a small amount of initial overshoot and ringing before reaching steady-state values. Overall, there is little difference between the B-S beam results and the reference solutions. The largest difference is $0.134 \%$ and occurs for $M_{k}$ in load case 8 . The differences between the steady-state and reference solutions for the non-redundant load cases are summarized in Table 4. Table 5 contains the corresponding results for the elastic-plastic material models. The FE results agree with the reference solutions to better than $1 \%$ in all cases. We conclude that the elastic regime responses for all DYNA3D beam element formulations are within reasonable agreement with the corresponding reference solutions. 


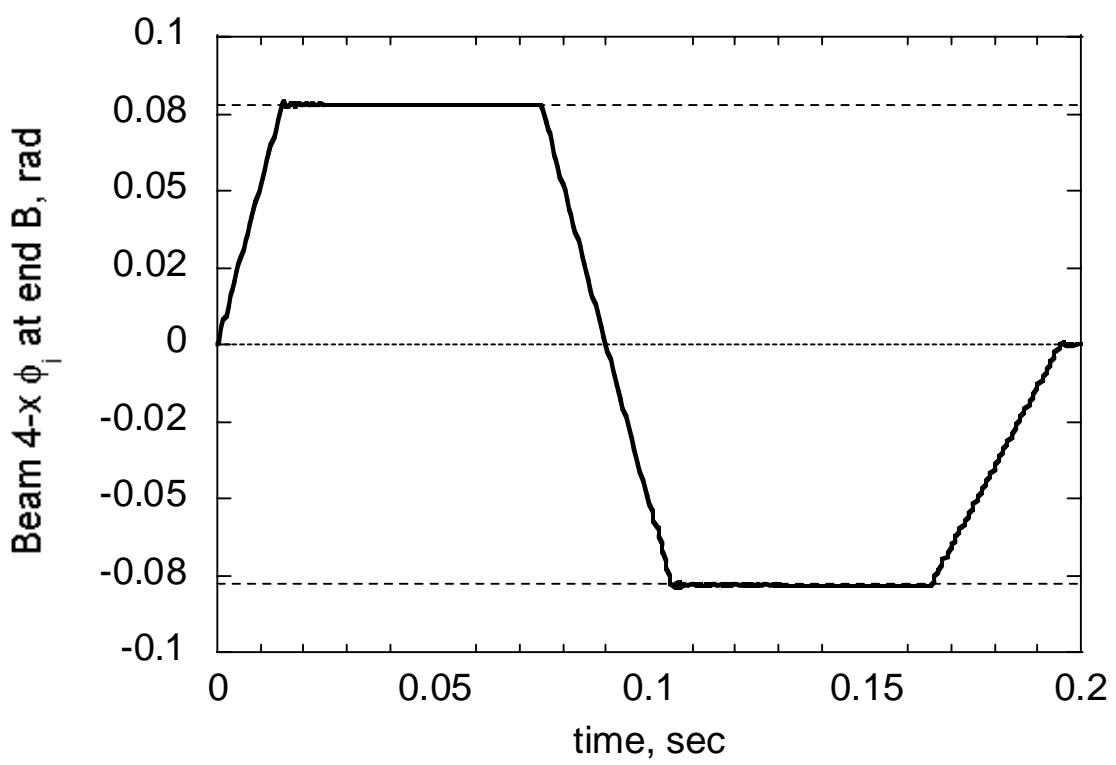

Figure 13. Rotation for beam 4-x at end B vs. time.

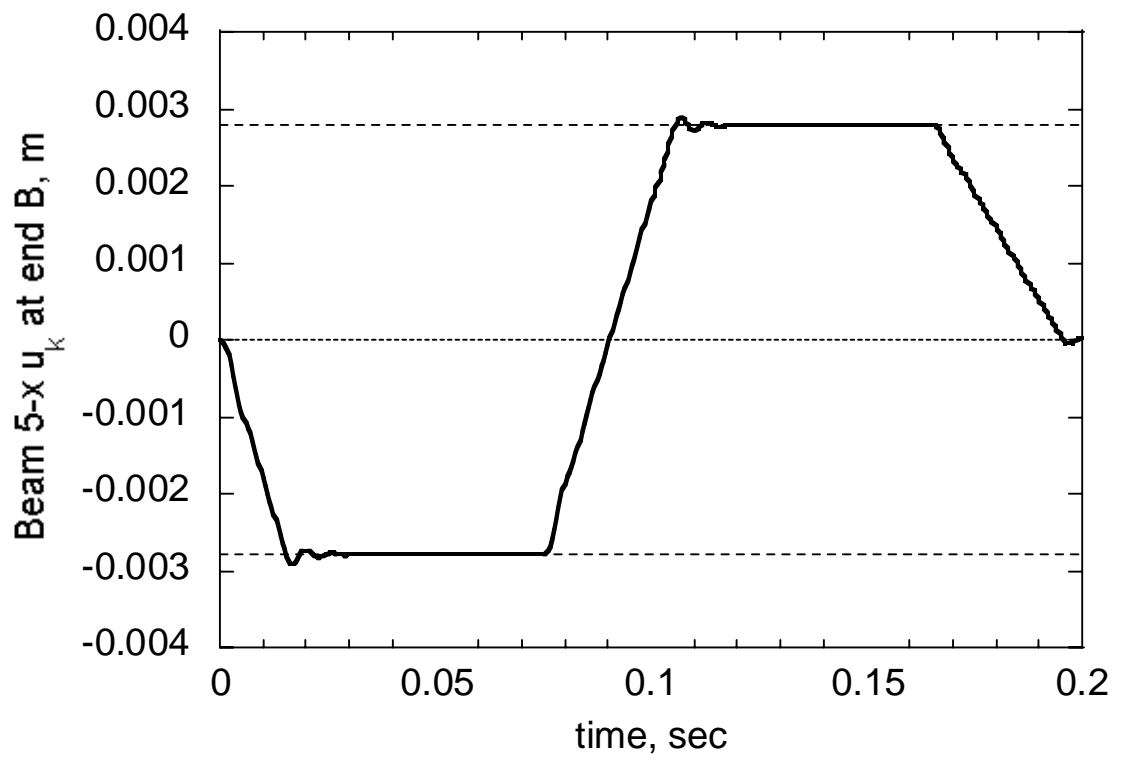

Figure 14. Transverse displacement for beam 5-x at end B vs. time. 


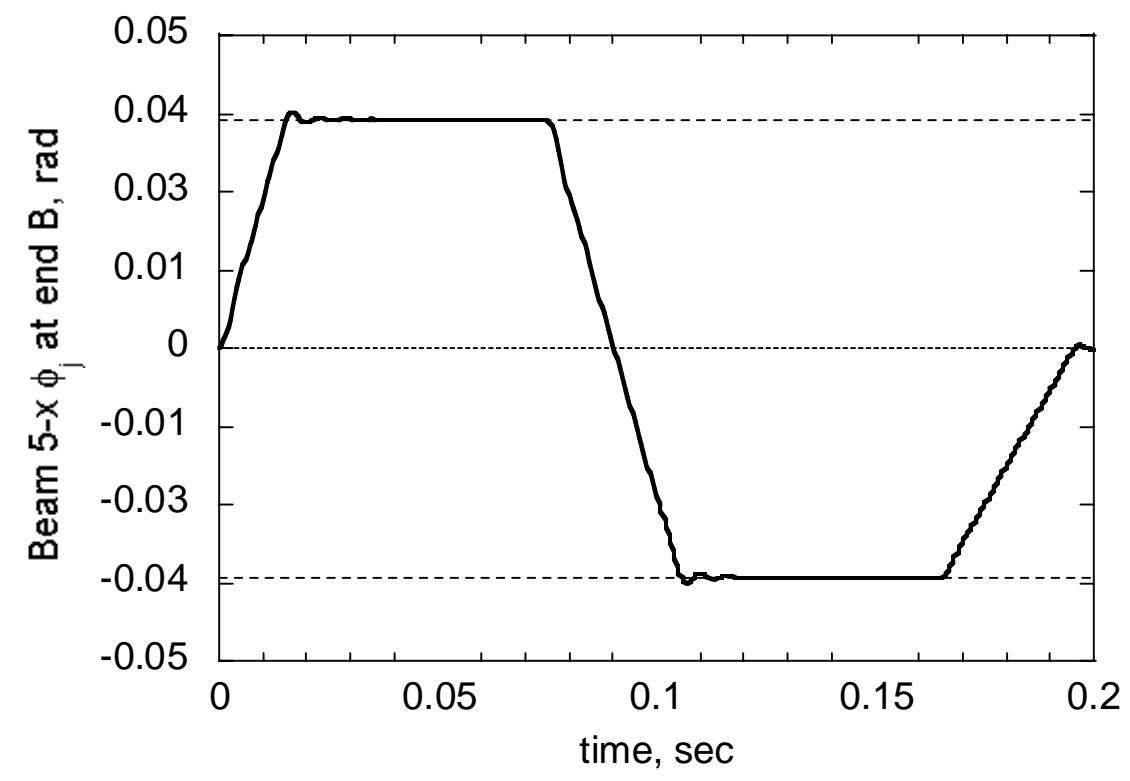

Figure 15. Rotation for beam 5-x at end B vs. time.

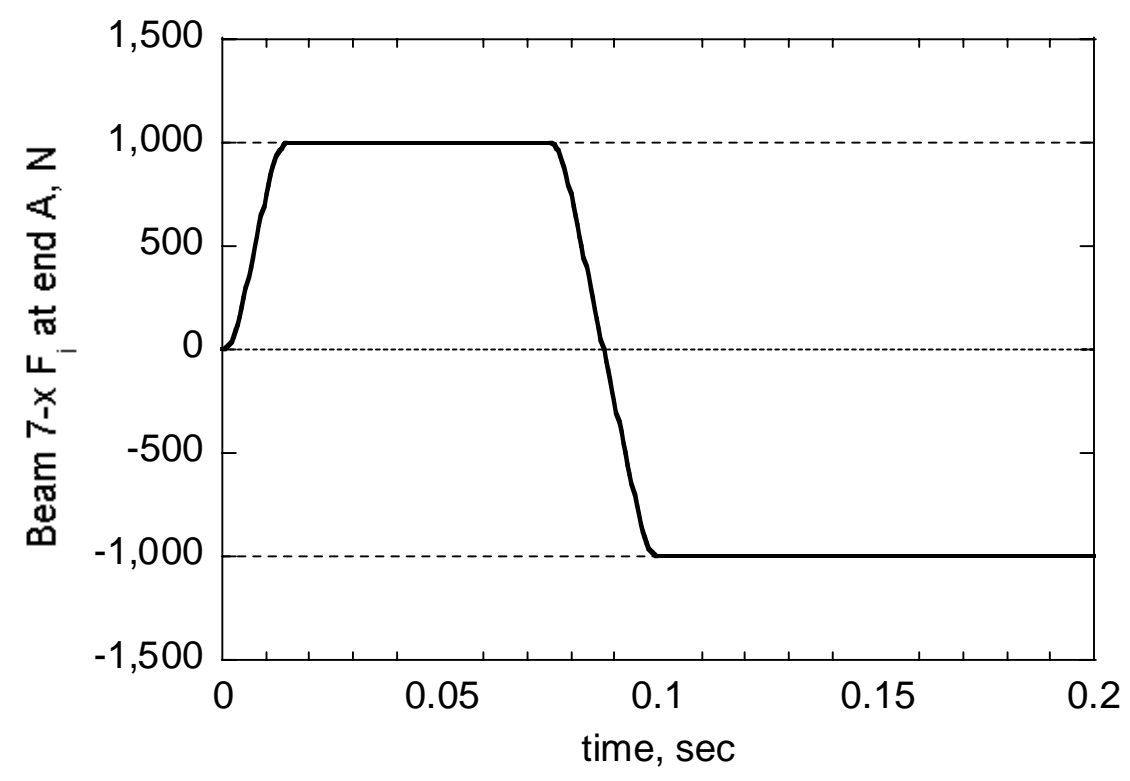

Figure 16. Axial force for beam 7-x vs. time. 


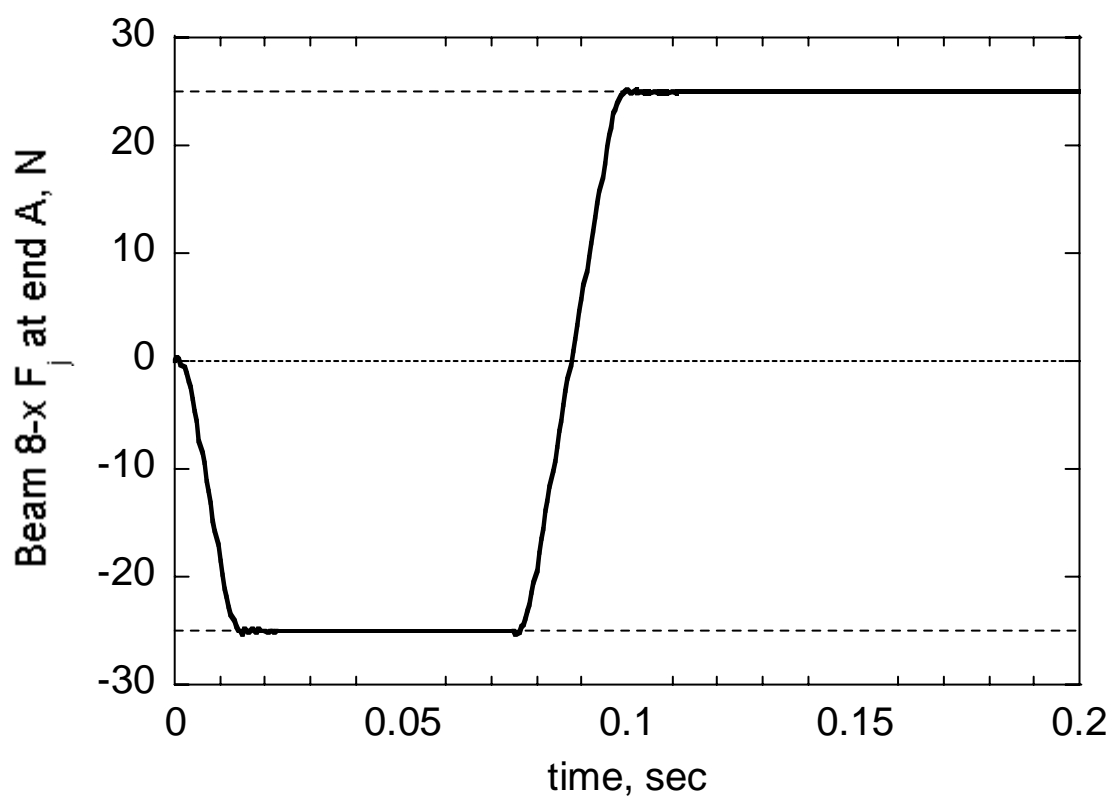

Figure 17. Transverse force for beam 8-x at end A vs. time.

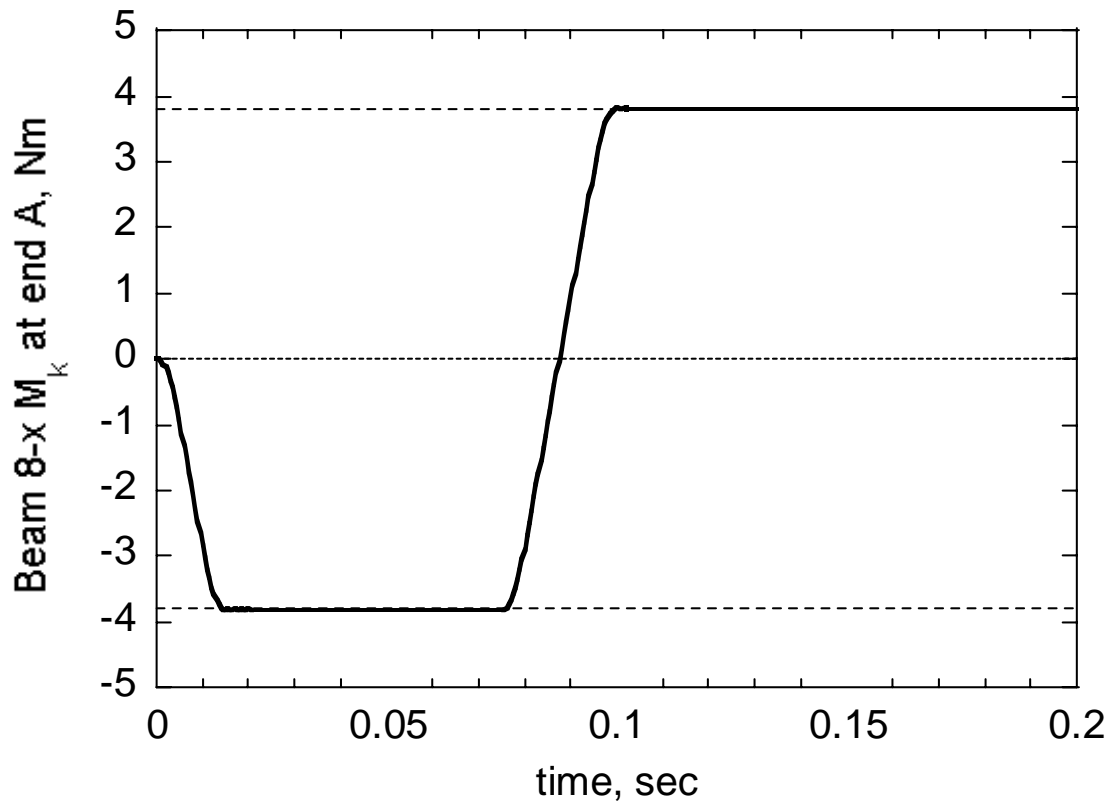

Figure 18. Bending moment for beam 8-x at end A vs. time. 


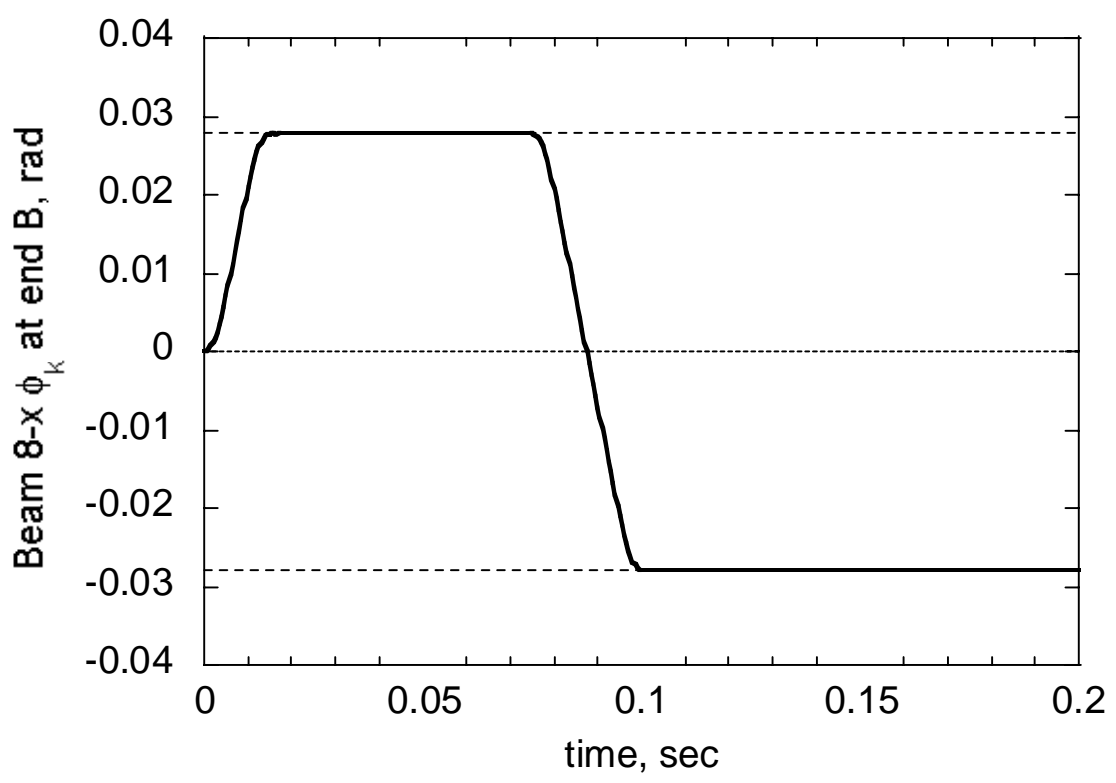

Figure 19. Rotation for beam 8-x at end B vs. time.

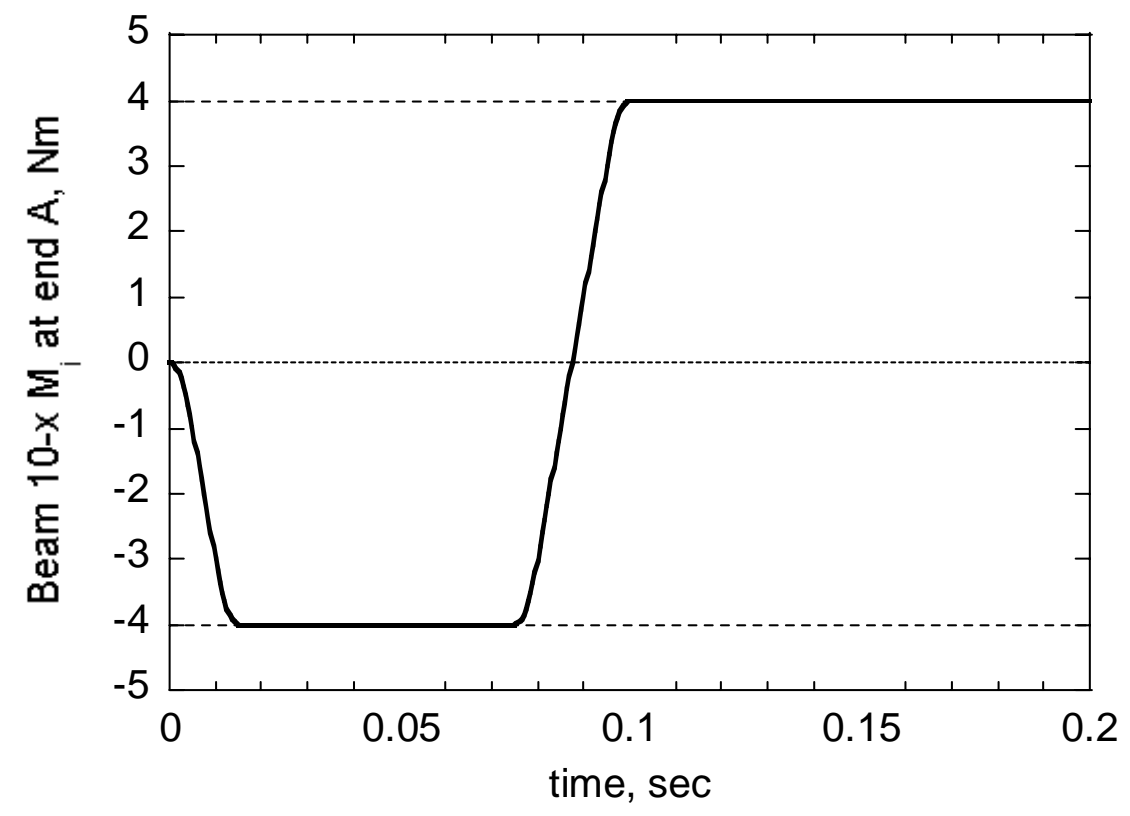

Figure 20. Bending moment for beam 10-x at end A vs. time. 


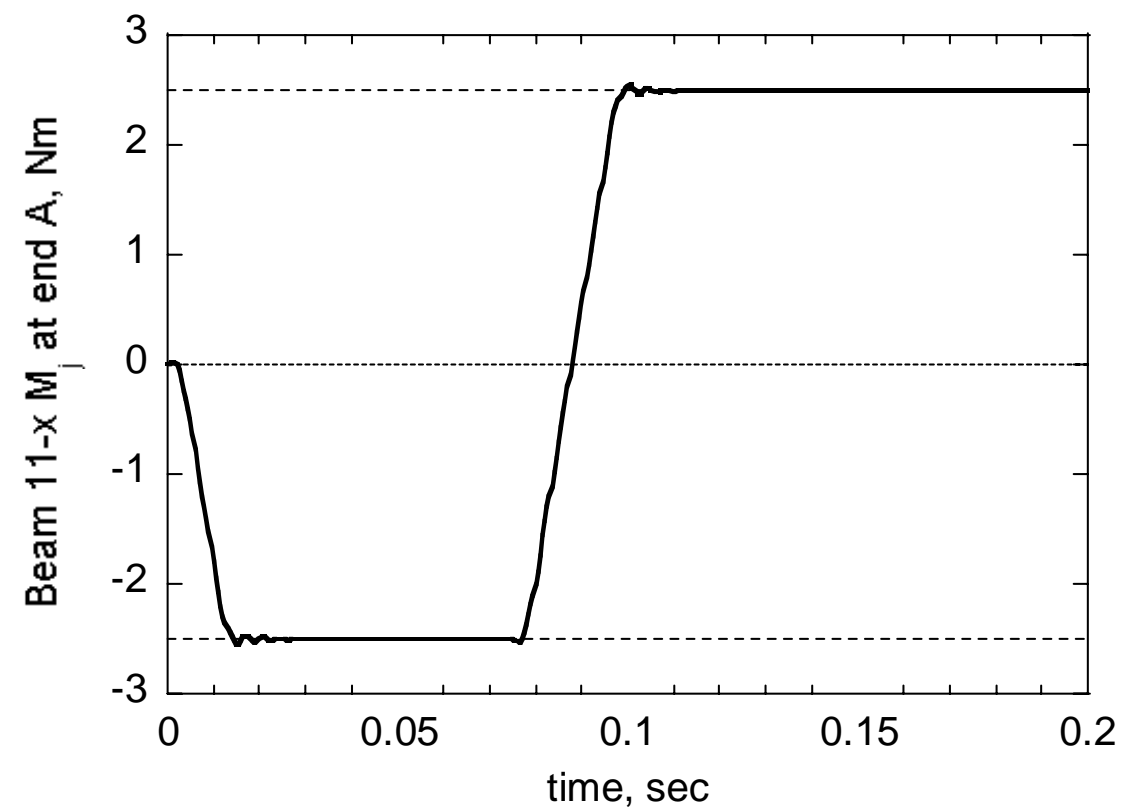

Figure 21. Bending moment for beam 11-x at end A vs. time.

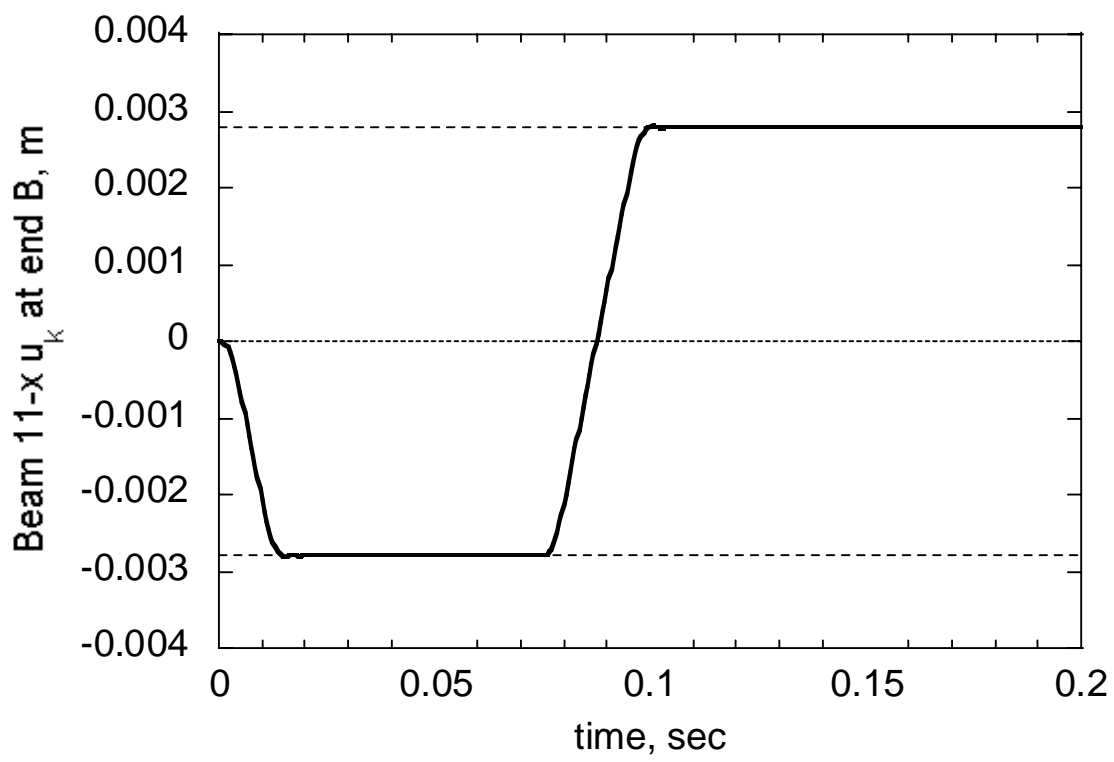

Figure 22. Transverse displacement for beam 11-x at end B vs. time. 
Table 4. Beam element results with elastic material model - expressed as percentage difference from reference solutions

\begin{tabular}{|c|c|c|c|c|c|c|c|}
\hline \hline Load case & Result verified & $B-S$ & $H-L 2$ & $H-L 3$ & $H-L 4$ & $H-L 5$ & $H-L 0$ \\
\hline \hline 1 & $u_{i}$ & 0.000 & 0.000 & 0.000 & 0.000 & 0.000 & 0.000 \\
\hline 2 & $u_{j}$ & 0.009 & 0.961 & 0.961 & 0.961 & 0.961 & 0.961 \\
\hline 2 & $\phi_{k}$ & 0.063 & 0.027 & 0.027 & 0.027 & 0.027 & 0.027 \\
\hline 4 & $\phi_{i}$ & 0.161 & 0.020 & 0.020 & 0.020 & 0.020 & 0.020 \\
\hline 5 & $u_{k}$ & 0.027 & 0.009 & 0.009 & 0.009 & 0.009 & 0.009 \\
\hline 5 & $\phi_{j}$ & 0.027 & 0.000 & 0.000 & 0.000 & 0.000 & 0.000 \\
\hline 7 & $F_{i}$ & 0.119 & 0.099 & 0.099 & 0.099 & 0.099 & 0.099 \\
\hline 8 & $F_{j}$ & 0.113 & 0.880 & 0.880 & 0.880 & 0.880 & 0.879 \\
\hline 8 & $M_{k}$ & 0.134 & 0.860 & 0.860 & 0.860 & 0.860 & 0.858 \\
\hline 8 & $\phi_{k}$ & 0.054 & 0.950 & 0.950 & 0.950 & 0.950 & 0.950 \\
\hline 10 & $M_{i}$ & 0.269 & 0.108 & 0.108 & 0.108 & 0.108 & 0.109 \\
\hline 11 & $M_{j}$ & 0.076 & 0.091 & 0.091 & 0.091 & 0.091 & 0.093 \\
\hline 11 & $u_{k}$ & 0.027 & 0.027 & 0.027 & 0.027 & 0.027 & 0.027 \\
\hline
\end{tabular}


Table 5. Beam element results with elastic-plastic material models (loaded in the elastic regime) expressed as percentage difference from reference solutions

\begin{tabular}{|c|c|c|c|c|c||}
\hline Load case & $\begin{array}{c}\text { Result } \\
\text { verified }\end{array}$ & $B$-S, material 3 & $B$-S, material 28 & H-L2, material 3 & H-L2, material 24 \\
\hline \hline 1 & $u_{i}$ & 0.000 & 0.000 & 0.000 & 0.000 \\
\hline 2 & $u_{j}$ & 0.009 & 0.009 & 0.961 & 0.961 \\
\hline 2 & $\phi_{k}$ & 0.063 & 0.063 & 0.027 & 0.027 \\
\hline 4 & $\phi_{i}$ & 0.161 & 0.161 & 0.020 & 0.020 \\
\hline 5 & $u_{k}$ & 0.027 & 0.027 & 0.009 & 0.009 \\
\hline 5 & $\phi_{j}$ & 0.027 & 0.027 & 0.000 & 0.000 \\
\hline 7 & $F_{i}$ & 0.119 & 0.119 & 0.099 & 0.099 \\
\hline 8 & $F_{j}$ & 0.113 & 0.113 & 0.880 & 0.880 \\
\hline 8 & $M_{k}$ & 0.134 & 0.134 & 0.860 & 0.860 \\
\hline 8 & $\phi_{k}$ & 0.054 & 0.054 & 0.950 & 0.108 \\
\hline 10 & $M_{i}$ & 0.269 & 0.269 & 0.108 & 0.091 \\
\hline 11 & $M_{j}$ & 0.076 & 0.076 & 0.091 & 0.027 \\
\hline 11 & $u_{k}$ & 0.027 & 0.027 & & \\
\hline \hline
\end{tabular}




\subsection{Plastic LOAding}

A group of problems is developed to verify the response of DYNA3D's beam elements in the plastic regime. Tension, bending, and torsion loading are considered. Because the element response depends heavily upon the numerical integration scheme, the plasticity study focuses on the H-L beam element formulation. To simplify the analyses and ensure stability of the solution, we apply translational and rotational displacements, in lieu of forces and moments, to generate the necessary loadings.

\subsubsection{Tension}

The beam problem considered for the tension-plasticity problem is identical to that used in the elastic analysis shown in Figure 1. End A remains completely fixed and an axial tensile displacement is applied to end B. In this analysis, only tensile deformation is considered because compression may lead to an elastic or a plastic buckling instability. The response is linearly elastic provided the displacement is less than the elastic limit given by:

$$
\delta_{e}=\sigma_{y} \frac{L}{E}
$$

For displacements beyond this limit, there is permanent (plastic) strain. Assuming a bi-linear stress-strain law, the axial load, $P$, at a displacement, $\delta$, that exceeds the elastic limit is given by:

$$
P=A_{c}\left\{E_{t}\left[\ln \left(\frac{\delta}{L}+1\right)-\varepsilon_{y}\right]+\sigma_{y}\right\}
$$

where $A_{c}, L$, and $\varepsilon_{y}$ are the cross sectional area of the beam, initial length of the beam, and yield strain, respectively. To verify the plasticity response of beam elements loaded in tension, we use the problem described above, with $\delta$ prescribed and $P$ monitored and compared to the reference solution given in Equation 7.

Two problem sets are established. In one, four five-element beam models are generated, each with a unique integration rule (H-LO, H-L2, H-L3, H-L4, and H-L5) and using material model 3 with the properties listed in Table 6. 3x3 Gauss quadrature is chosen for the user-defined integration rule. Note that $\beta=1.0$ is also specified for this material model, although in this situation it is inconsequential to the results. The second problem set is identical, except that material model 24 is used with the same properties.

For each of the problem sets, a final displacement of $\delta=0.00211 \mathrm{~m}$ is applied at end B; from Equation 7, this displacement corresponds to an axial load of $6875 \mathrm{~N}$. As with the elastic 
problem sets, the displacement is attained by prescribing a nodal velocity through a load curve that is similar in shape to Figure 7 but with an expanded time scale. To ensure that the response is not influenced by dynamic effects, the duration of the analysis is set to 0.3 seconds. Mass-

Table 6. Material properties for tension-plasticity problems

\begin{tabular}{|c|c|c|c|c|c|c|}
\hline Material model & $E, \mathrm{GPa}$ & $v$ & $\rho, \mathrm{kg} / \mathrm{m}^{3}$ & $\sigma_{y}, \mathrm{MPa}$ & $E_{t}, \mathrm{GPa}$ & $\beta$ \\
\hline \hline 3 & 200 & 0.3 & 7860 & 250 & 2 & 1 \\
\hline 24 & 200 & 0.3 & 7860 & 250 & 2 & N/A \\
\hline
\end{tabular}

proportional damping is not required. Figure 23 shows the axial force as a function of time in the H-L2 beam with material model 3. The dashed line in Figure 23 is the reference solution. The observed axial load is within $0.010 \%$ of the reference solution. Simulations are completed for all cases in the two problem sets. The results are numerically equivalent for all integration rules and material models.

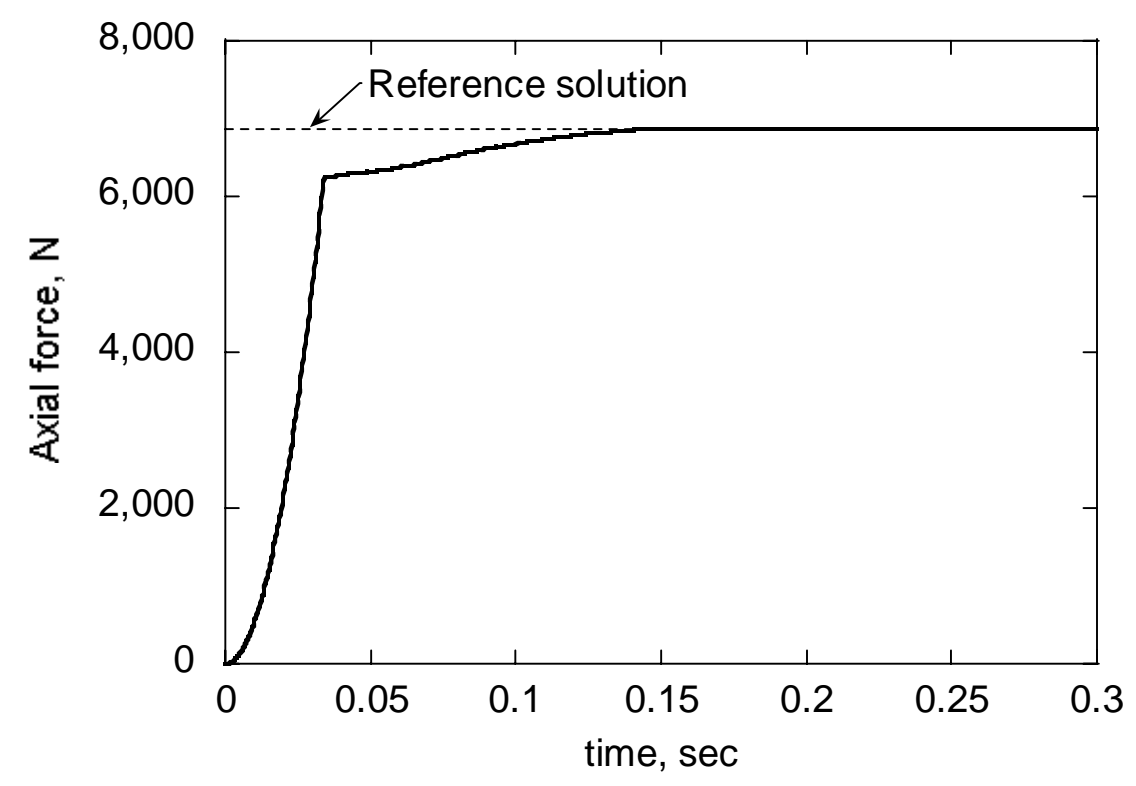

Figure 23. Axial force vs. time for loadings that probe the plastic response

\subsubsection{Bending}

In the second group of plasticity problems, a rotational displacement about the local $k$-axis is applied to end $\mathrm{B}$ of the cantilevered beam to create a condition of pure bending. Assuming an elastic-perfectly plastic material model, the fully plastic limit moment, $M_{p}$, is given by [10]: 


$$
M_{p}=\frac{b h^{2} \sigma_{y}}{4}
$$

for a rectangular cross-section with width $b$ and height $h$. Since this is an asymptotic limit, the magnitude of the applied rotational displacement must be chosen to ensure that the model is very near the fully plastic limit moment.

Reaching strain levels associated with $M_{p}$ would require extreme deflection for a multi-element model, such that the beam would wrap around the $k$-axis multiple times. Hence, a single-element beam model is used for this analysis. End A is held fixed and a rotational displacement of 1.25 rad $\left(72^{\circ}\right)$ is applied to end $\mathrm{B}$. (The rotational displacement is achieved by specifying a rotational velocity through an appropriate load curve.)

Two problem sets are established. The first includes four single-element beams, each with a unique integration rule (H-L0, H-L2, H-L3, H-L4, and H-L5) using material model 3 and the elastic perfectly-plastic properties listed in Table 7. In this analysis, a square cross-section beam with $b=h=0.005 \mathrm{~m}$ and $\sigma_{y}=250 \mathrm{MPa}$ are assumed, corresponding to a reference solution of $M_{p}=7.81 \mathrm{Nm}$. The second problem set is identical except that material model 24 is specified. 2x200 trapezoidal quadrature is chosen for the user-defined integration rule. The 200 integration points are evenly distributed and aligned parallel with the local element s-axis (for a definition of element axes, see [1]). This unusual numerical integration rule was chosen in an attempt to accurately calculate the fully-plastic bending limit.

Table 7. Material properties for bending, torsion and tension-torsion plasticity problems

\begin{tabular}{|c|c|c|c|c|c|c||}
\hline $\begin{array}{c}\text { Material } \\
\text { model }\end{array}$ & $E, \mathrm{GPa}$ & $v$ & $\rho, \mathrm{kg} / \mathrm{m}^{3}$ & $\begin{array}{c}\sigma_{y}, \\
\mathrm{MPa}\end{array}$ & $E_{t}, \mathrm{GPa}$ & $\beta$ \\
\hline \hline 3 & 200 & 0.3 & 7860 & 250 & $\approx 0$ & 1 \\
\hline 24 & 200 & 0.3 & 7860 & 250 & $\approx 0$ & N/A \\
\hline
\end{tabular}

Figure 24 shows the recorded moment about the local element t-axis as a function of time for the various numerical integration rules used in conjunction with material model 3. The response for all integration rules is identical in the elastic regime. Thereafter, the response is heavily dependent on the numerical integration rule specified, i.e., the placement of integration points. Table 8 gives the difference between the DYNA3D steady-state and the reference solution bending results. The 2x200 trapezoidal user-defined integration rule gives the most accurate result, due to the high number of integration points near the neutral axis. While this integration rule gives an accurate result, it significantly extends run-time and is, therefore, somewhat impractical for common use. The results are numerically identical for material types 3 and 24 . 


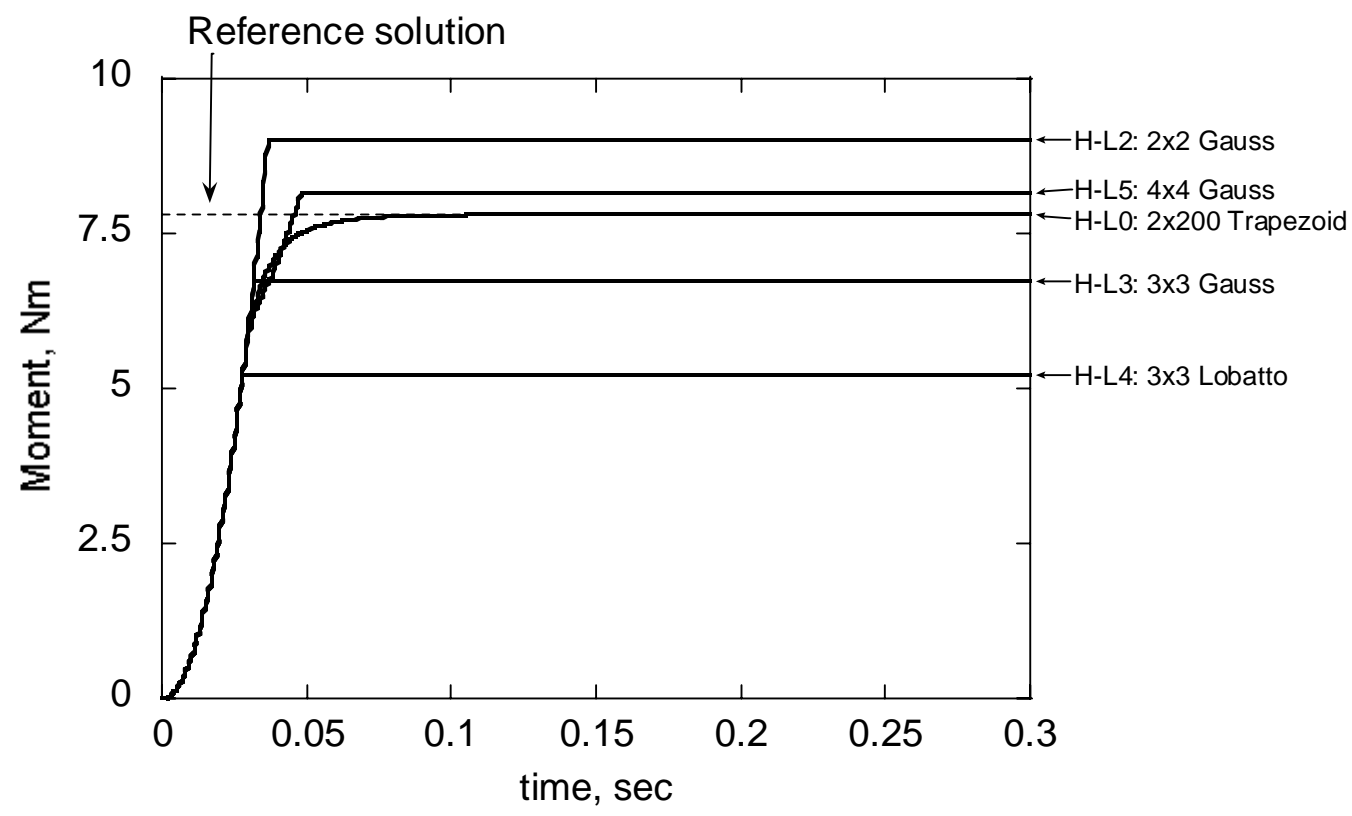

Figure 24. Bending moment vs. time for $\mathrm{H}-\mathrm{L}$ beam elements with various numerical integration rules. Loading exceeds the elastic limit and approaches the fully plastic limit.

Table 8. Beam bending results for fully plastic moment - expressed as percentage difference from reference solution

\begin{tabular}{|c|c|}
\hline Element type & \% difference \\
\hline \hline $\begin{array}{c}\text { H-LO: } 2 \times 200 \\
\text { Trapezoidal }\end{array}$ & 0.025 \\
\hline H-L2 & 15.655 \\
\hline H-L3 & 13.796 \\
\hline H-L4 & 33.228 \\
\hline H-L5 & 4.420 \\
\hline
\end{tabular}




\subsubsection{Torsion}

In the third group of plasticity problems, a torsional displacement is applied at end B with end A held fixed to create a condition of pure shear. For an elastic-perfectly plastic material model with shear yield strength $\tau_{y}$, the fully plastic limit torque, $T_{p}$, is given by [10]:

$$
T_{p}=\frac{2 \pi r^{3} \tau_{y}}{3}
$$

for a solid circular cross-section with radius $r$. This is an asymptotic limit; hence the magnitude of the applied torsional displacement is chosen to ensure that the result is very near the fullyplastic limit.

Single-element beam models are subjected to torsional displacements of $1.25 \mathrm{rad}\left(72^{\circ}\right)$ at end B and fixed in translation and rotation at end A. The magnitude of the displacement is chosen such that the beams approach their fully-plastic limit torque, $T_{p}$. The beam has a solid circular crosssection, with $r=2.821 \mathrm{~mm}$. Assuming $\tau_{y}=\sigma_{y} / \sqrt{3}=144.3 \mathrm{MPa}, T_{p}=6.78 \mathrm{Nm}$. As with the tension and bending analyses, all standard H-L integration rules are explored, including the userdefined integration scheme shown in Figure 25. Both material models 3 and 24 are used, with the elastic perfectly-plastic material properties shown in Table 7. Both material models gives numerically identical results. Figure 26 shows the torque in each beam element as a function of time, along with the reference solution. The result for the H-LO user-defined integration rule is within $0.860 \%$ of the reference solution. The results for the remaining element variants are within $6.157 \%$ of the reference solution. Again, the accuracy of the numerical results appears to be highly dependent upon the integration rule utilized.

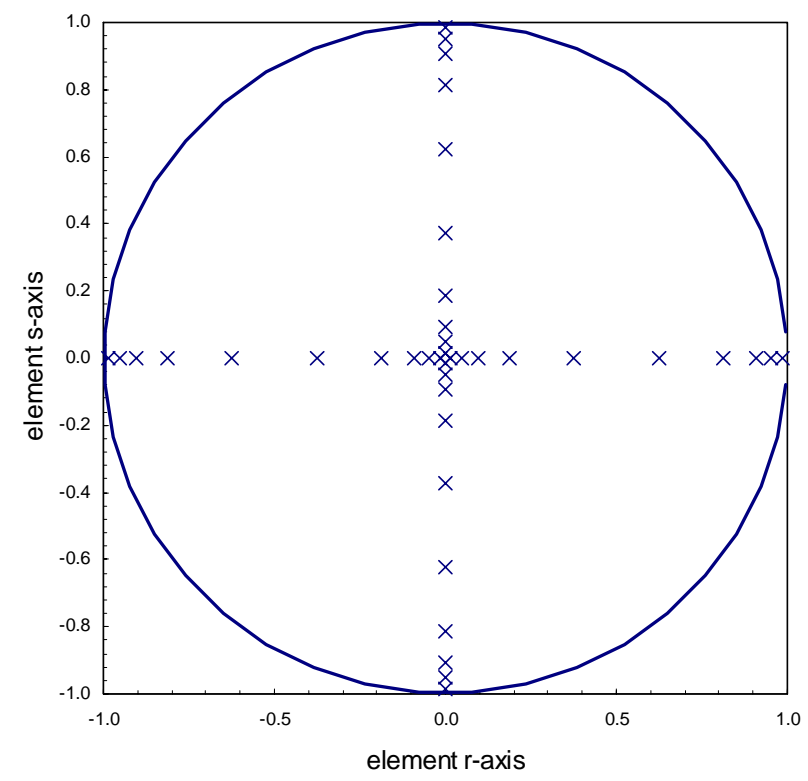

Figure 25. Numerical integration coordinates for torsional plasticity problem. $\mathrm{x}$-marks indicate integration points. 


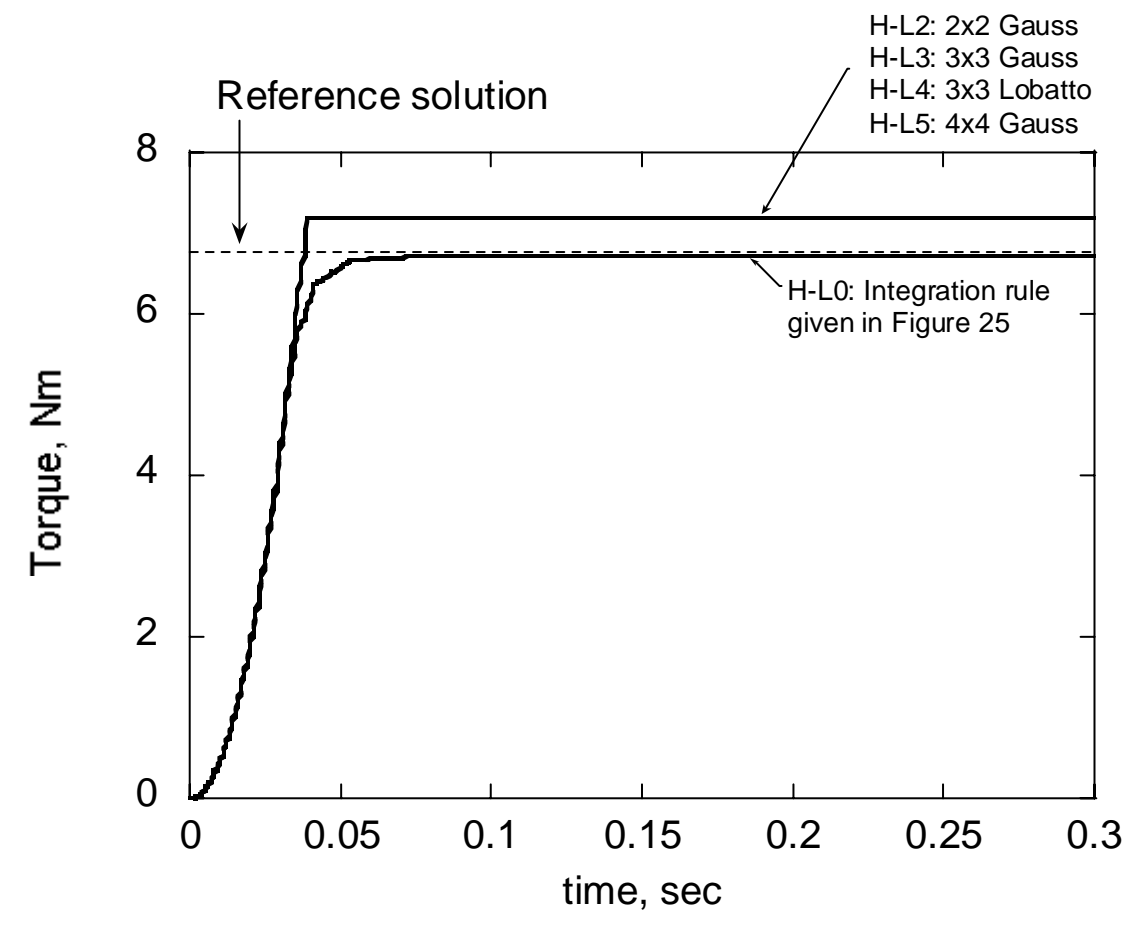

Figure 26. Torque vs. time for loading to the plastic limit torque

\subsubsection{Thin-walled tube with Tension / Torsion Loading}

In the final plastic deformation verification problems, a thin-walled tube geometry, shown in Figure 27, is subjected to several strain history paths involving axial and torsional displacements. The thin-walled hollow tube geometry is chosen for its clearly-defined shear and normal stress states for the given displacements. The outer and inner radii are $R=5 \mathrm{~mm}$ and $r=4.5 \mathrm{~mm}$, respectively, and the beam length is $L=0.1525 \mathrm{~m}$. The radii are selected such that their ratio, $r / R=0.9$, is consistent with the assumptions of thin-walled tube analysis.

Six separate load cases are generated in which single-loading-mode (SL) strain histories are prescribed. In these analyses, end A (Figure 27) is held fixed, and the appropriate translation/rotation is applied to end B. In the first three cases, labeled SL-1, -2, and -3 in Table 9 , axial displacements corresponding to strains of $0.5 \varepsilon_{y}, \varepsilon_{y}$, and $10 \varepsilon_{y}$ are imposed. In the second three cases, labeled SL $-4,-5$, and -6 in Table 9, torsional displacements corresponding to strains of $0.5 \gamma_{y}, \gamma_{y}$, and $10 \gamma_{y}$ are imposed. In all six cases, the effective plastic strain, $\varepsilon_{e}^{p}$, is obtained from the DYNA3D results. $\varepsilon_{e}^{p}$ is given by:

$$
\varepsilon_{e}^{p}=\int d \varepsilon_{e}^{p}
$$


where $d \varepsilon_{e}^{p}$ is the effective plastic strain increment, as calculated from the plastic strain tensor, $d \varepsilon_{i j}^{p}:$

$$
d \varepsilon_{e}^{p}=\left[\frac{2}{3} d \varepsilon_{i j}^{p} d \varepsilon_{i j}^{p}\right]^{1 / 2}
$$

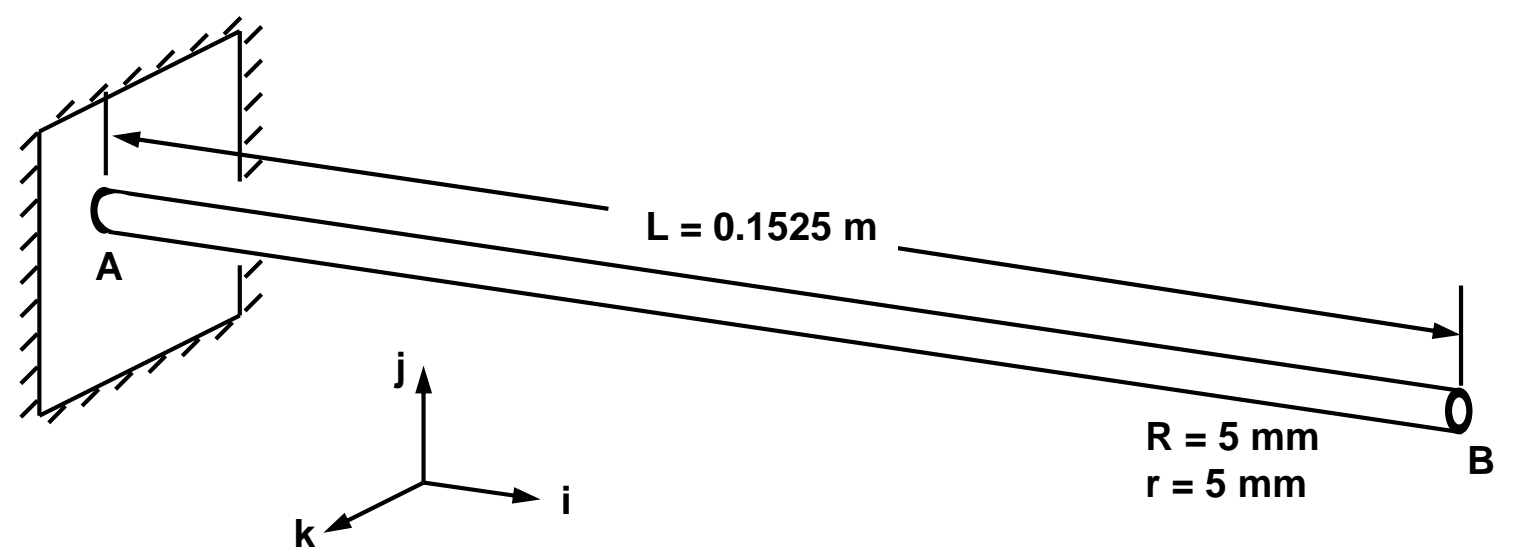

Figure 27. Thin-walled tube geometry for tension-torsion plasticity problems

Table 9. Single loading (SL) mode cases, reference solution and results

\begin{tabular}{|c|c|c|c|c|c|c|}
\hline Load case & $\begin{array}{c}\text { Total axial } \\
\text { strain, } \varepsilon_{x}\end{array}$ & $\begin{array}{c}\text { Plastic axial } \\
\text { strain, } \varepsilon_{x}^{p}\end{array}$ & $\begin{array}{c}\text { Total shear } \\
\text { strain, } \gamma_{x}\end{array}$ & $\begin{array}{c}\text { Plastic shear } \\
\text { strain, } \gamma_{x}^{p}\end{array}$ & $\begin{array}{c}\text { Theoretical } \\
\text { effective } \\
\text { plastic } \\
\text { strain, } \varepsilon_{e}^{p}\end{array}$ & $\begin{array}{c}\text { Observed } \\
\text { effective } \\
\text { plastic } \\
\text { strain, } \varepsilon_{e}^{p}\end{array}$ \\
\hline SL-1 & $0.5 \varepsilon_{y}$ & 0 & 0 & 0 & 0 & 0 \\
\hline SL -2 & $\varepsilon_{y}$ & 0 & 0 & 0 & 0 & 0 \\
\hline SL -3 & $10 \varepsilon_{y}$ & $9 \varepsilon_{y}$ & 0 & 0 & $9 \varepsilon_{y}$ & $8.9 \varepsilon_{y}$ \\
\hline SL -4 & 0 & 0 & $0.5 \gamma_{y}$ & 0 & 0 & 0 \\
\hline SL -5 & 0 & 0 & $\gamma_{y}$ & 0 & 0 & 0 \\
\hline SL -6 & 0 & 0 & $10 \gamma_{y}$ & $9 \gamma_{y}$ & $5.2 \gamma_{y}$ & $4.7 \gamma_{y}$ \\
\hline
\end{tabular}


For pure axial loading with plastic component $\varepsilon_{x}^{p}, d \varepsilon_{i j}^{p}$ is given by [10]:

$$
d \varepsilon_{i j}^{p}=\left[\begin{array}{ccc}
d \varepsilon_{x}^{p} & 0 & 0 \\
0 & \frac{-d \varepsilon_{x}^{p}}{2} & 0 \\
0 & 0 & \frac{-d \varepsilon_{x}^{p}}{2}
\end{array}\right]
$$

such that $\varepsilon_{e}^{p}=\varepsilon_{x}^{p}$, where $x$ is the major axis of the beam.

For pure torsional loading with plastic component $\gamma_{x}^{p}, d \varepsilon_{i j}^{p}$ is given by [10]:

$$
d \varepsilon_{i j}^{p}=\left[\begin{array}{ccc}
0 & \frac{d \gamma_{x}^{p}}{2} & 0 \\
\frac{d \gamma_{x}^{p}}{2} & 0 & 0 \\
0 & 0 & 0
\end{array}\right]
$$

leading to $\varepsilon_{e}^{p}=\frac{\sqrt{3}}{3} \gamma_{x}^{p} \approx 0.58 \gamma_{x}^{p}$. Hence, for $\gamma_{x}^{p}=10 \gamma_{y}-\gamma_{y}=9 \gamma_{y}, \varepsilon_{e}^{p}=\gamma_{y}$.

For these load cases, a model using a single beam element with user-defined numerical integration (H-L0) is defined. The placement of integration points, shown in Figure 28, is chosen to provide adequate resolution of the stress state within a reasonable computation time. The weight assigned to each integration point is the fractional area of the segment surrounding the integration point divided by the cross-sectional area of the hollow tube. In the analysis, end A is held fixed, and the appropriate translation/rotation is applied to end B. Material model 24 is employed with the properties listed in Table 7. The corresponding yield strains are $\varepsilon_{y}=0.00125$ and $\gamma_{y}=0.00192$.

Table 9 gives a summary of results for the various load cases. For a pure axial displacement, the observed effective plastic strains agree well with the theoretical results. In the case of a pure torsional displacement, the result is approximately $10 \%$ lower than the reference solution. This difference is likely due to the fact that the theoretical result applies to an infinitesimally thin tube, whereas the DYNA3D result accounts for the finite thickness of the tube. 


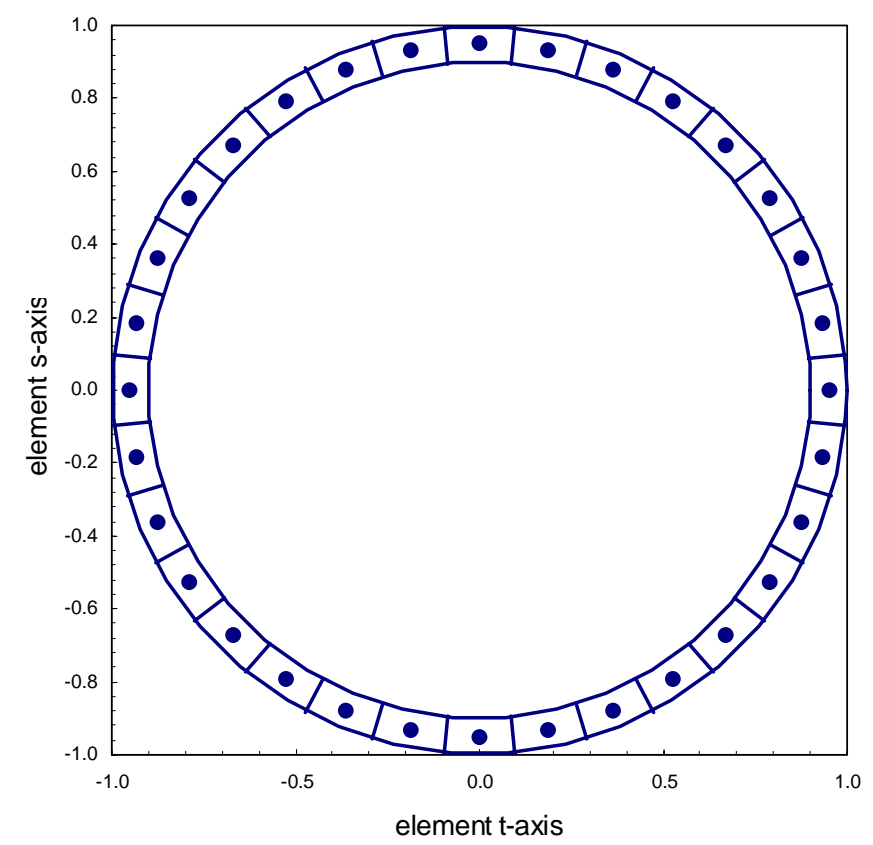

Figure 28. Numerical integration coordinates for thin-walled hollow tube tension / torsion plasticity problem

\subsection{UNI-AXIAL ELASTIC DEFORMATION - PARALLEL PROBLEM}

Large-scale FE simulations can be accommodated by the massively-parallel version of DYNA3D, ParaDyn [11]. In ParaDyn, a FE model is subdivided into smaller partitions, with the solution for each partition being computed by CPU nodes working in parallel. To facilitate the analysis, information is passed between nodes regarding the status of partition solutions at each time step.

A basic problem suitable for verifying these parallel aspects is developed in which a straight beam of length $L$, cross-sectional area $A$ and elastic modulus $E$ is fixed at one end and an axial displacement, $\delta$, is applied to the other end. The reference solution for axial force, $P$, is given by [6]:

$$
P=\frac{A_{c} E}{L} \delta
$$

A FE model of a single beam consisting of 500 collinear 1-mm-long B-S elements is developed to simulate the problem. The beam cross-section is square with $A_{c}=2.5 \times 10^{-5} \mathrm{~m}^{2}$. The material is elastic, with $E=200 \mathrm{GPa}$, and $v=0.3$. For the current problem, $\delta=3.05 \times 10^{-5} \mathrm{~m}$, and the reference solution is $P=305 \mathrm{~N}$. To reduce the serial DYNA3D run time to approximately one 
minute while allowing the axial force to equilibrate, the density is specified as $\rho=2 \times 10^{7} \mathrm{~kg} / \mathrm{m}^{3}$ much higher than realistic engineering materials. To attenuate vibrations in the resulting stress wave, mass-proportional damping with $\xi=1.0$ and $\omega_{n}=270 \mathrm{rad} / \mathrm{sec}$ is applied. The serial DYNA3D result is in perfect agreement with the reference solution. Results of the ParaDyn verification analyses will be reported in the future.

\section{TRUSS ELEMENT VERIFICATION}

To model structural members that carry axial load without shear, bending, or torque, DYNA3D incorporates standard truss elements and H-L degenerate beams (H-L1). Problem sets are developed to verify the elastic and elastic-plastic responses of these elements.

Regarding the elastic response, three problem sets are developed, one each for material models 1 , 3 and 24. The forces, displacements, load curves, material properties, geometric parameters, damping, and reference solutions are numerically equivalent to load cases 1 and 7 (Tables 1 and 2), respectively, used in the analogous axial deformation, elastic response, beam element problem sets. In each problem set, twelve single-element truss models are included: two element variants with two load conditions (force and displacement) in three orientations (x, $\mathrm{y}$, and $\mathrm{z}$ ). In all cases, the results are numerically equivalent for both element variants and all material models and orientations. Figures 29 and 30 show the results for an applied force and displacement, respectively, along with the corresponding reference solutions (dashed lines) for loading in the elastic regime. For the applied force problem, the observed displacement is within $0.010 \%$ of the reference solution, and for the applied displacement problem, the observed force is within $0.009 \%$ of the reference solution.

For verification of the truss element plastic response, two problem sets are developed, one each for material models 3 and 24. In each problem set, two single-element truss models are established, one for each element variant, both oriented in the x-direction. The applied displacements, load curve, material properties, geometric parameters, and reference solutions are equivalent to the tension-plasticity beam element problem sets described in Section 2.5.1. Figure 31 shows the axial force vs. time, along with the corresponding reference solution. The observed axial force is within $0.006 \%$ of the reference solution. 


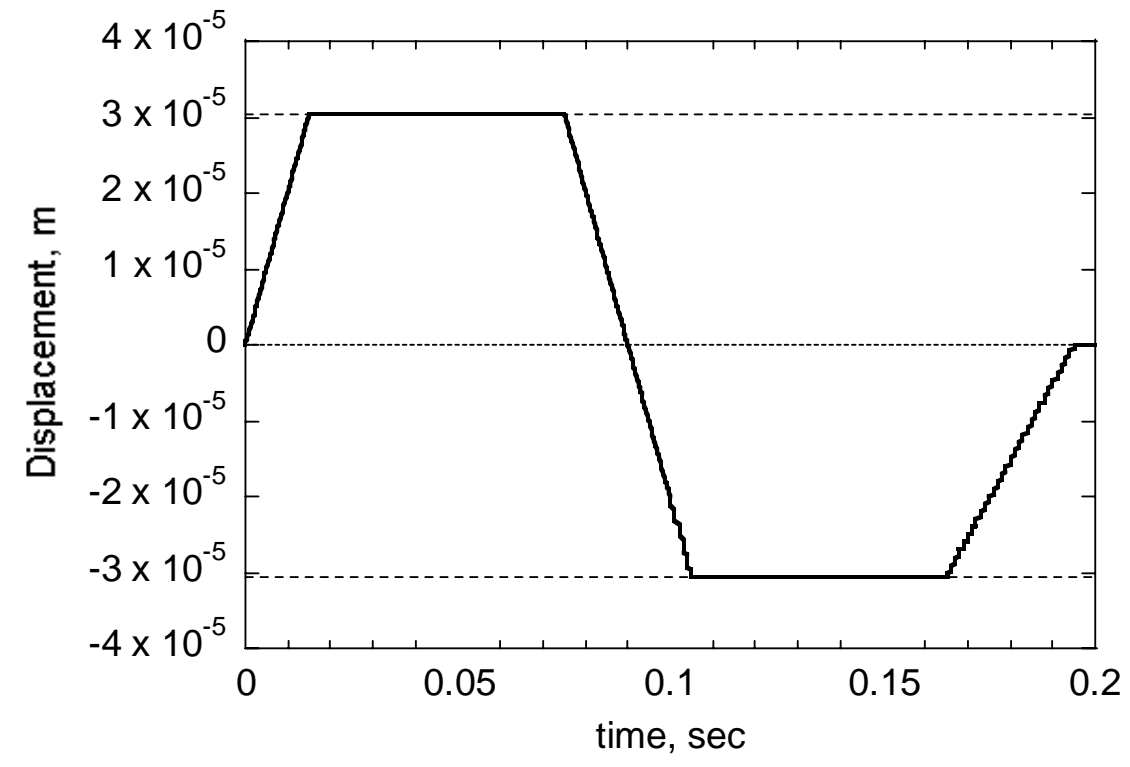

Figure 29. Axial displacement vs. time for truss elements

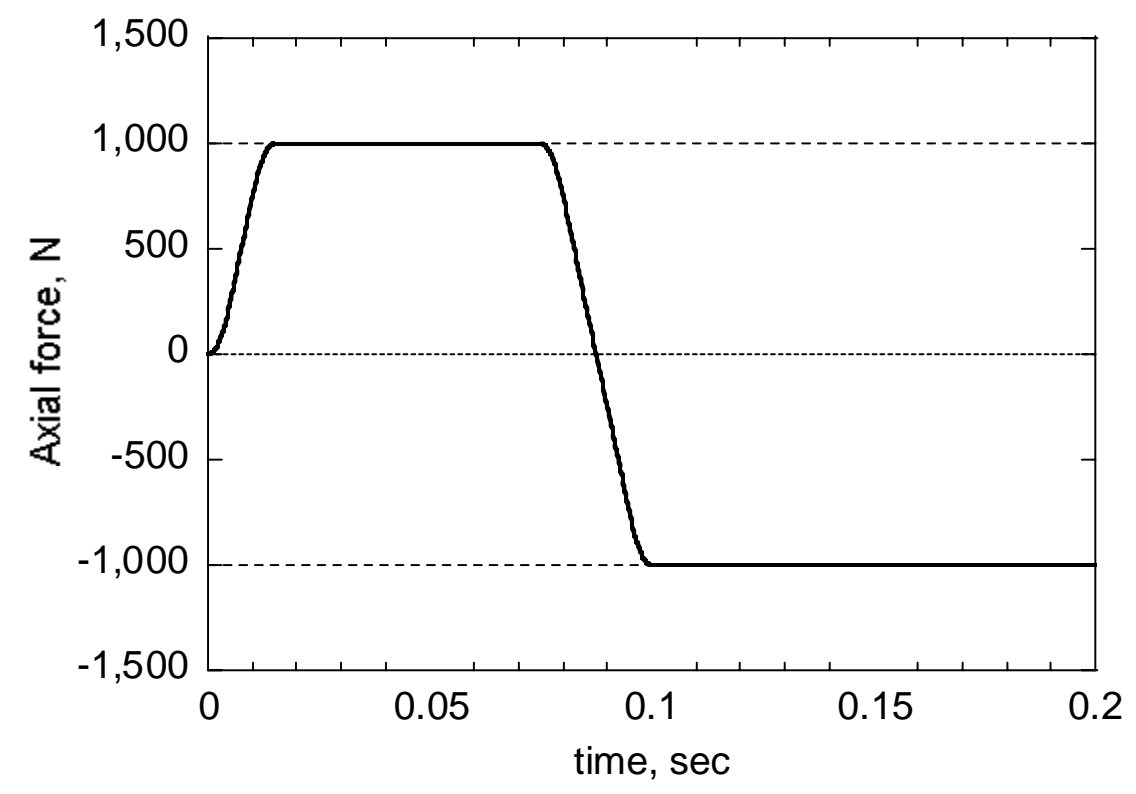

Figure 30. Axial force vs. time for truss elements 


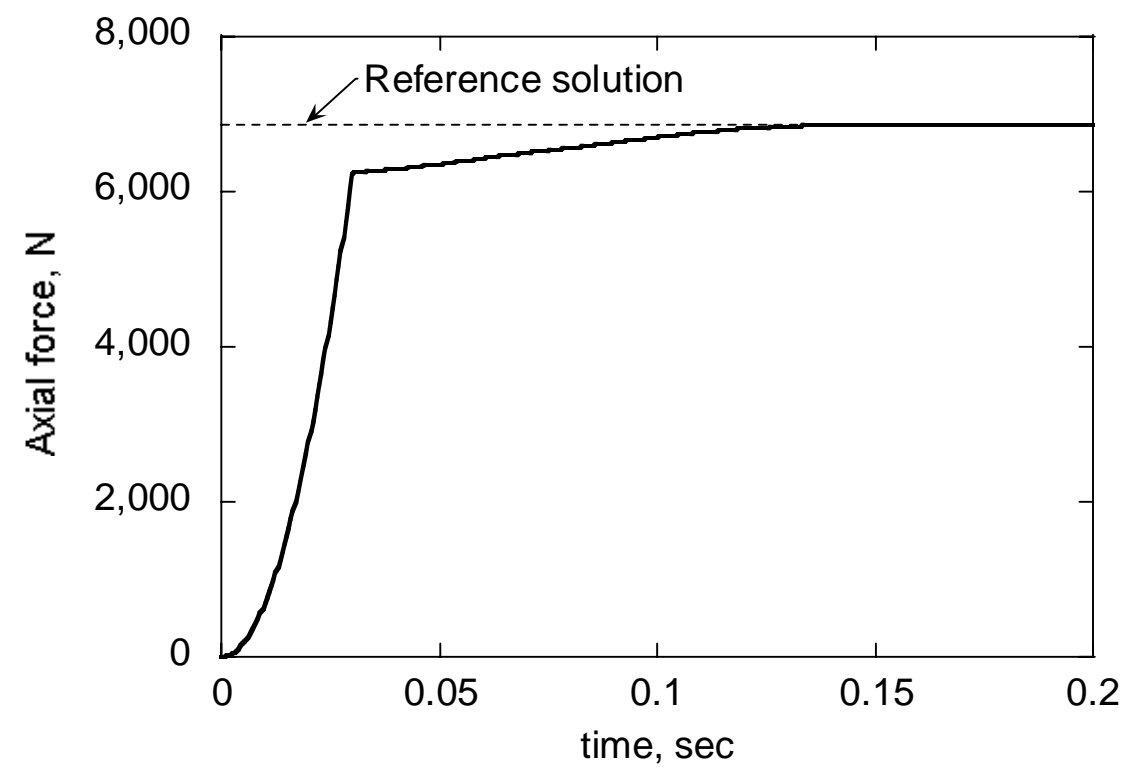

Figure 31. Axial force vs. time for truss elements - plastic loading

\section{CONCLUSIONS}

Verification problem sets have been generated for beam and truss elements in DYNA3D and the results compared with analytical reference solutions. For loading in the elastic regime, all DYNA3D results are within $1 \%$ of the corresponding reference solutions, providing confidence in their performance in this domain. For purely tensile loading that exceeds the elastic limit, the observed DYNA3D results are within 1\% of the reference solution. For H-L beam elements loaded in the plastic regime via bending, the results are heavily dependent on the type of integration rule specified. The most accurate results, with errors of about $0.02 \%$, are obtained with a user-defined numerical integration rule with a high degree of in-plane resolution. In the case of H-L beam elements loaded in torsion into the plastic regime, the results are consistent for all standard numerical integration rules. Once again, a user-defined numerical integration rule that is refined near the center axis provided the most accurate results, with an error of $0.86 \%$. Truss elements are also verified to provide accurate results (within $0.01 \%$ ) for elastic and elasticplastic loading. 


\section{REFERENCES}

1. Lin, J.I., "DYNA3D: A Nonlinear, Explicit, Three-Dimensional Finite Element Code for Solid and Structural Mechanics, User Manual” UCRL-MA-107254, June, 2005.

2. Hughes, T.J.R., and Liu, W.K., "Nonlinear Finite Element Analysis of Shells: Part I. Threedimensional shells,” Computer Methods in Applied Mechanics and Engineering, 26, 331-362, 1981.

3. Hughes, T.J.R., and Liu, W.K., "Nonlinear Finite Element Analysis of Shells: Part II. Twodimensional shells,” Computer Methods in Applied Mechanics and Engineering, 27, 167-181, 1981.

4. Belytschko, T., and Hsieh, B.J., "Non-linear Transient Finite Element Analysis with Convected Co-ordinates," International Journal for Numerical Methods in Engineering, 7, 255271, 1973.

5. Belytschko, T., and Schwer, L., "Large Displacement, Transient Analysis of Space Frames," International Journal for Numerical Methods in Engineering, 11, 65-84, 1977.

6. Beer, F.P. and Johnston, E.R., Mechanics of Materials, McGraw-Hill Book Company, New York, 1981.

7. TrueGrid Manual, Version 2.1.0, XYZ Scientific Applications, Inc., 2001.

8. Speck, D.E., “GRIZ Finite Element Analysis Results Visualization for Unstructured Grids, User Manual,” UCRL-MA-115696, Rev. 2, November, 2001.

9. Rao, S.S., Mechanical Vibrations, Addison-Wesley Publishing Company, 1986.

10. Mendelson, A., Plasticity-Theory and Application, Macmillan, New York, NY, 1968.

11. Hoover, C.G., De Groot, A.J., and Sherwood, R.J., "ParaDyn: A Parallel Nonlinear, Explicit, Three-Dimensional Finite Element Code for Solid and Structural Mechanics, User Manual” UCRL-MA-140943-revised, January, 2004. 


\section{APPENDIX}

Sample TrueGrid input deck for beam element linear elastic verification.

title Dyna3D Beam Verification Analysis

dyna3d

C **************** Dyna3D Options ****************

dynaopts term 0.2 prti 0.02 plti 0.02 rfpf 1 ;

c ********* Material declarations for Hughes-Liu beam element $* * * * * * * * *$

c Mat_ID Mat_Type

dynamats 11 rho 7860

beam elfom hl

shear 1.0 quad -1 bmcross 0 sloc 0 tloc 0

sthi 0.005 tthi 0.005

e $2 \mathrm{e}+11$ pr 0.33 ;

C ******** Material declarations for Belytschko-Schwer beam element

$* * * * * * * *$

c Properties commented out to implement H-L elements

c Rectangular cross-section beam properties:

c dynamats 11 rho 7860

c beam elfom bs carea 2.5e-05

c iss $5.21 \mathrm{e}-11$ itt $5.21 \mathrm{e}-11$ irr $1.04 \mathrm{e}-10$ sarea $2.5 \mathrm{e}-05$

c e $2.0 \mathrm{e}+11$ pr 0.33 ;

c ******** Hughes-Liu user-defined integration (3x3 Gauss)

bind 11.0

$\begin{array}{lll}-0.77459667 & -0.77459667 & 0.30864197\end{array}$

$\begin{array}{llll}-0.77459667 & 0.00000000 & 0.49382716\end{array}$

$\begin{array}{lll}-0.77459667 & 0.77459667 & 0.30864197\end{array}$

$0.00000000-0.774596670 .49382716$

$\begin{array}{llll}0.00000000 & 0.00000000 & 0.79012346\end{array}$

$\begin{array}{llll}0.00000000 & 0.77459667 & 0.49382716\end{array}$

$\begin{array}{llll}0.77459667 & -0.77459667 & 0.30864197\end{array}$

$\begin{array}{llll}0.77459667 & 0.00000000 & 0.49382716\end{array}$

$\begin{array}{llll}0.77459667 & 0.77459667 & 0.30864197\end{array}$

;

C ******** Load curve definitions

lcd 1 lp 000.01510 .07510 .105 -1 0.165 -1 0.195010 ;

lcd 2 lp 000.00510 .01010 .01500 .07500 .080 -1 0.095 -1 0.1010 ; 
lcd 3 lp 001 0;

C $* * * * * * * * * * * * * * *$ Beam 1-X $* * * * * * * * * * * * * * *$ block 1 6;1 2;1 2; 0 0.1525;0 0.1525;0 0.1525;

ibm $111211111 \mathrm{j} 1$;

b $111111 \mathrm{dx} 1 \mathrm{dy} 1 \mathrm{dz} 1 \mathrm{rx} 1$ ry 1 rz 1 ;

fc 21121111000100

mate 0

endpart

C $* * * * * * * * * * * * * * *$ Beam 2-x *****************

block 1 6;1 2;1 2; 0 0.1525;0 0.1525;0.1 0.2525;

ibm $111211111 \mathrm{j} 1$;

b $111111 \mathrm{dx} 1 \mathrm{dy} 1 \mathrm{dz} 1 \mathrm{rx} 1$ ry $1 \mathrm{rz} 1$;

fc 211211125010

mate 0

endpart

C **************** Beam 3-x ****************

block 1 6;1 2;1 2; 0 0.1525;0 0.1525;0.2 0.3525;

ibm $111211111 \mathrm{j} 1$;

b $111111 \mathrm{dx} 1 \mathrm{dy} 1 \mathrm{dz} 1 \mathrm{rx} 1 \mathrm{ry} 1 \mathrm{rz} 1$;

fc 211211125001

mate 0

endpart

C $* * * * * * * * * * * * * * *$ Beam $4-\mathrm{x} * * * * * * * * * * * * * * *$

block 1 6;1 2;1 2; 0 0.1525;0 0.1525;0.3 0.4525;

ibm $111211111 \mathrm{j} 1$;

b $111111 \mathrm{dx} 1 \mathrm{dy} 1 \mathrm{dz} 1 \mathrm{rx} 1 \mathrm{ry} 1 \mathrm{rz} 1$;

mom $21121114 \mathrm{x}$

mate 0

endpart

C $* * * * * * * * * * * * * * *$ Beam 5-X $* * * * * * * * * * * * * * *$

block 1 6;1 2;1 2; 0 0.1525;0 0.1525;0.4 0.5525;

ibm $111211111 \mathrm{j} 1$;

b $111111 \mathrm{dx} 1 \mathrm{dy} 1 \mathrm{dz} 1 \mathrm{rx} 1$ ry $1 \mathrm{rz} 1$;

mom $21121112.5 \mathrm{y}$

mate 0

endpart

C **************** Beam 6-x $* * * * * * * * * * * * * * *$

block 1 6;1 2;1 2; 0 0.1525;0 0.1525;0.5 0.6525;

ibm $111211111 \mathrm{j} 1$;

b $111111 \mathrm{dx} 1 \mathrm{dy} 1 \mathrm{dz} 1 \mathrm{rx} 1$ ry 1 rz 1 ; 
mom 21121112.5 z

mate 0

endpart

C **************** Beam 7-x ***************

block 1 6;1 2;1 2; 0 0.1525;0 0.1525;0.6 0.7525;

ibm $111211111 \mathrm{j} 1$;

b 111111 dy $1 \mathrm{dz} 1 \mathrm{rx} 1 \mathrm{ry} 1 \mathrm{rz} 1$;

fv 21121120.00305100 ;

fv $11111131.0 \quad 100$;

mate 0

endpart

C **************** Beam 8-x ****************

block 1 6;1 2;1 2; 0 0.1525;0 0.1525;0.7 0.8525;

ibm $111211111 \mathrm{j} 1$;

b $111111 \mathrm{dx} 1 \mathrm{dz} 1 \mathrm{rx} 1$ ry 1 ;

fv 21121120.2836010 ;

fv 11111131.0010 ;

mate 0

endpart

C $* * * * * * * * * * * * * * *$ Beam 9-x ***************

block 1 6;1 2;1 2; 0 0.1525;0 0.1525;0.8 0.9525;

ibm $111211111 \mathrm{j} 1$;

b $111111 \mathrm{dx} 1 \mathrm{dy} 1 \mathrm{rx} 1 \mathrm{rz} 1$;

fv 21121120.2836001

fv $11111131.0 \quad 001$;

mate 0

endpart

$\mathrm{C} * * * * * * * * * * * * * * *$ Beam 10- $\mathrm{x} * * * * * * * * * * * * * * *$

block 1 6;1 2;1 2; 0 0.1525;0 0.1525;0.9 1.0525;

ibm $111211111 \mathrm{j} 1$;

b $111111 \mathrm{dx} 1 \mathrm{dy} 1 \mathrm{dz} 1 \mathrm{ry} 1 \mathrm{rz} 1$;

frb 21121127.8 dofv x ;

mate 0

endpart

C *************** Beam 11-x ***************

block 1 6;1 2;1 2; 0 0.1525;0 0.1525;1.0 1.1525;

ibm $111211111 \mathrm{j} 1$;

b $111111 \mathrm{dx} 1$ dy $1 \mathrm{rx} 1 \mathrm{rz} 1$;

frb 21121123.66 dofv y ;

fv 11111131.0001 ;

mate 0 
endpart

C *************** Beam 12-x ****************

block 1 6;1 2;1 2; 0 0.1525;0 0.1525;1.1 1.2525;

ibm $111211111 \mathrm{j} 1$;

b $111111 \mathrm{dx} 1 \mathrm{dz} 1 \mathrm{rx} 1$ ry 1 ;

frb 21121123.66 dofv z ;

fv $11111131.0 \quad 010$;

mate 0

endpart

C ***************** Beam 1-y *****************

block 1 2;1 6 ;1 2; 0 0.1525; 0.1525 0.305; 0 0.1525;

jbm $111121111 \mathrm{k} 1$;

b $111111 \mathrm{dx} 1 \mathrm{dy} 1 \mathrm{dz} 1 \mathrm{rx} 1$ ry $1 \mathrm{rz} 1$;

fc 12112111000010

mate 0

endpart

C **************** Beam 2-y ****************

block 1 2;1 6;1 2; 0.1525 0.305;0.1525 0.305; 0 0.1525;

jbm $111121111 \mathrm{k} 1$;

b $111111 \mathrm{dx} 1 \mathrm{dy} 1 \mathrm{dz} 1 \mathrm{rx} 1$ ry $1 \mathrm{rz} 1$;

fc 121121125001

mate 0

endpart

C $* * * * * * * * * * * * * * *$ Beam 3-y $* * * * * * * * * * * * * * *$

block 1 2;1 6;1 2; 0.305 0.4575; 0.1525 0.305; 0 0.1525;

jbm $111121111 \mathrm{k} 1$;

b $111111 \mathrm{dx} 1 \mathrm{dy} 1 \mathrm{dz} 1 \mathrm{rx} 1$ ry $1 \mathrm{rz} 1$;

fc 121121125100

mate 0

endpart

C **************** Beam 4-y *****************

block 1 2;1 6 ; 1 2; 0.4575 0.61;0.1525 0.305;0 0.1525;

jbm $111121111 \mathrm{k} 1$;

b $111111 \mathrm{dx} 1 \mathrm{dy} 1 \mathrm{dz} 1 \mathrm{rx} 1$ ry $1 \mathrm{rz} 1$;

mom $12112114 \mathrm{y}$

mate 0

endpart

C **************** Beam 5-y ****************

block 1 2;1 6;1 2; 0.61 0.7625; 0.1525 0.305; 0 0.1525;

jbm $111121111 \mathrm{k} 1$; 
b $111111 \mathrm{dx} 1 \mathrm{dy} 1 \mathrm{dz} 1 \mathrm{rx} 1$ ry $1 \mathrm{rz} 1$;

mom $12112112.5 \mathrm{z}$

mate 0

endpart

C **************** Beam 6-y ****************

block 1 2;1 6;1 2; 0.76250 .915 ; 0.1525 0.305; 0 0.1525;

jbm $111121111 \mathrm{k} 1$;

b $111111 \mathrm{dx} 1 \mathrm{dy} 1 \mathrm{dz} 1 \mathrm{rx} 1$ ry $1 \mathrm{rz} 1$;

mom $12112112.5 \mathrm{x}$

mate 0

endpart

C **************** Beam 7-y ****************

block 1 2;1 6 ;1 2; 0.915 1.0675; 0.15250 .305 ; 0 0.1525;

jbm $111121111 \mathrm{k} \mathrm{1}$;

b $111111 \mathrm{dx} 1 \mathrm{dz} 1 \mathrm{rx} 1 \mathrm{ry} 1 \mathrm{rz} 1$;

fv 12112120.00305010 ;

fv $11111131.0 \quad 010$;

mate 0

endpart

C **************** Beam 8-y ****************

block 1 2;1 6;1 2; 1.06751 .22 ; 0.1525 0.305; 0 0.1525;

jbm $111121111 \mathrm{k} 1$;

b $111111 \mathrm{dx} 1$ dy 1 ry $1 \mathrm{rz} 1$;

fv 12112120.2836001 ;

fv $11111131.0 \quad 001$;

mate 0

endpart

C **************** Beam 9-y ****************

block 1 2;1 6;1 2; 1.22 1.3725; 0.1525 0.305; 0 0.1525;

jbm $111121111 \mathrm{k} 1$;

b 111111 dy $1 \mathrm{dz} 1$ rx 1 ry 1 ;

fv 12112120.2836100 ;

fv 11111131.0100 ;

mate 0

endpart

C **************** Beam 10-y ****************

block 1 2;1 6 ;1 2; 1.3725 1.525;0.1525 0.305;0 0.1525;

jbm $111121111 \mathrm{k} 1$;

b $111111 \mathrm{dx} 1 \mathrm{dy} 1 \mathrm{dz} 1 \mathrm{rx} 1 \mathrm{rz} 1$;

frb 12112127.8 dofv y ;

mate 0 
endpart

C **************** Beam 11-y ***************

block 1 2;1 6;1 2; 1.5251 .6775 ; 0.1525 0.305; 0 0.1525;

jbm $111121111 \mathrm{k} 1$;

b 111111 dy $1 \mathrm{dz} 1 \mathrm{rx} 1$ ry 1 ;

frb 12112123.66 dofv z ;

fv $11111131.0 \quad 100$;

mate 0

endpart

C $* * * * * * * * * * * * * * *$ Beam 12-y $* * * * * * * * * * * * * * *$

block 1 2;1 6;1 2; 1.67751 .83 ; 0.1525 0.305; 0 0.1525;

jbm $111121111 \mathrm{k} 1$;

b $111111 \mathrm{dx} 1$ dy 1 ry 1 rz 1 ;

frb 12112123.66 dofv x ;

fv $11111131.0 \quad 001$;

mate 0

endpart

C *************** Beam 1-z

block 1 2;1 2;1 6 ; 0 0.1525;-0.1525 0; 0 0.1525;

kbm 111112111 i 1 ;

b $111111 \mathrm{dx} 1 \mathrm{dy} 1 \mathrm{dz} 1 \mathrm{rx} 1$ ry $1 \mathrm{rz} 1$;

fc 11211211000001

mate 0

endpart

C *************** Beam 2-z $* * * * * * * * * * * * * * *$

block 1 2;1 2;1 6; 0 0.1525;-0.305 -0.1525; 0 0.1525;

kbm 111112111 i 1 ;

b $111111 \mathrm{dx} 1 \mathrm{dy} 1 \mathrm{dz} 1 \mathrm{rx} 1$ ry $1 \mathrm{rz} 1$;

fc 112112125100

mate 0

endpart

C $* * * * * * * * * * * * * * *$ Beam 3-z $* * * * * * * * * * * * * * *$

block 1 2;1 2;1 6; 0 0.1525;-0.4575 -0.305; 0 0.1525;

kbm 111112111 i 1 ;

b $111111 \mathrm{dx} 1 \mathrm{dy} 1 \mathrm{dz} 1 \mathrm{rx} 1$ ry 1 rz 1 ;

fc 112112125010

mate 0

endpart

C $* * * * * * * * * * * * * * *$ Beam 4-z

block 1 2;1 2;1 6 ; 0 0.1525;-0.61 -0.4575; 0 0.1525; 
kbm 111112111 i 1 ;

b $111111 \mathrm{dx} 1 \mathrm{dy} 1 \mathrm{dz} 1 \mathrm{rx} 1$ ry $1 \mathrm{rz} 1$;

mom $11211214 \mathrm{z}$

mate 0

endpart

C *************** Beam 5-z $* * * * * * * * * * * * * * *$

block 1 2;1 2;1 6; 0 0.1525;-0.7625 -0.61; 0 0.1525;

kbm 111112111 i 1 ;

b $111111 \mathrm{dx} 1 \mathrm{dy} 1 \mathrm{dz} 1 \mathrm{rx} 1 \mathrm{ry} 1 \mathrm{rz} 1$;

mom $11211212.5 \mathrm{x}$

mate 0

endpart

C $* * * * * * * * * * * * * * *$ Beam 6-z $* * * * * * * * * * * * * * *$

block 1 2;1 2;1 6; 0 0.1525;-0.915 -0.7625; 0 0.1525;

kbm 111112111 i 1 ;

b $111111 \mathrm{dx} 1 \mathrm{dy} 1 \mathrm{dz} 1 \mathrm{rx} 1 \mathrm{ry} 1 \mathrm{rz} 1$;

mom $11211212.5 \mathrm{y}$

mate 0

endpart

C **************** Beam 7-z ***************

block 1 2;1 2;1 6 ; 0 0.1525;-1.0675 -0.915; 0 0.1525;

kbm 111112111 i 1 ;

b $111111 \mathrm{dx} 1 \mathrm{dy} 1 \mathrm{rx} 1$ ry $1 \mathrm{rz} 1$;

fv 11211220.00305001 ;

fv $11111131.0 \quad 001$;

mate 0

endpart

C $* * * * * * * * * * * * * * *$ Beam 8-z $* * * * * * * * * * * * * * *$

block 1 2;1 2;1 6; 0 0.1525;-1.22 -1.0675; 0 0.1525;

kbm 111112111 i 1 ;

b $111111 \mathrm{dy} 1 \mathrm{dz} 1 \mathrm{rx} 1 \mathrm{rz} 1$;

fv 11211220.2836100 ;

fv 11111131.0100 ;

mate 0

endpart

c $* * * * * * * * * * * * * * *$ Beam 9-z $* * * * * * * * * * * * * * *$

block 1 2;1 2;1 6; 0 0.1525;-1.3725 -1.22; 0 0.1525;

kbm 111112111 i 1 ;

b $111111 \mathrm{dx} 1 \mathrm{dz} 1 \mathrm{ry} 1 \mathrm{rz} 1$;

fv 11211220.2836010 ;

fv $11111131.0 \quad 010$; 


\section{mate 0}

endpart

$\mathrm{C} * * * * * * * * * * * * * * *$ Beam 10-z

block 1 2;1 2;1 6 ; 0 0.1525;-1.525 -1.3725; 0 0.1525;

kbm 111112111 i 1 ;

b $111111 \mathrm{dx} 1 \mathrm{dy} 1 \mathrm{dz} 1 \mathrm{rx} 1$ ry 1 ;

frb 11211227.8 dofv z ;

mate 0

endpart

C **************** Beam 11-z ****************

block 1 2;1 2;1 6; 0 0.1525;-1.6775 -1.525; 0 0.1525;

kbm 111112111 i 1 ;

b $111111 \mathrm{dx} 1 \mathrm{dz} 1$ ry $1 \mathrm{rz} 1$;

frb 11211223.66 dofv x ;

fv 11111131.0010 ;

mate 0

endpart

C $* * * * * * * * * * * * * * *$ Beam 12-z $* * * * * * * * * * * * * * *$

block 1 2;1 2;1 6; 0 0.1525;-1.83 -1.6775; 0 0.1525;

kbm 111112111 i 1 ;

b $111111 \mathrm{dy} 1 \mathrm{dz} 1 \mathrm{rx} 1 \mathrm{rz} 1$;

frb 11211223.66 dofv y ;

fv 11111131.0100 ;

mate 0

endpart

merge

stp .001

c interrupt

cont 